\title{
Radiocarbon geochronology of the sediments of the São Paulo Bight (southern Brazilian upper margin)
}

\author{
MICHEL M. MAHIQUES, SILVIA H.M. SOUSA, LETICIA BURONE, RENATA H. NAGAI, \\ ILSON C.A. SILVEIRA, RUBENS C.L. FIGUEIRA, RAFAEL G. SOUTELINO, \\ LEANDRO PONSONI and DANIEL A. KLEIN
}

Instituto Oceanográfico da Universidade de São Paulo, Praça do Oceanográfico, 191, 05508-120 São Paulo, SP, Brasil

Manuscript received on October 14, 2009; accepted for publication on October 14, 2010

\begin{abstract}
The aim of this work was to generate an inventory of the data on radiocarbon datings obtained from sediments of the São Paulo Bight (southern Brazilian upper margin) and to analyze the data in terms of Late Quaternary sedimentary processes and sedimentation rates. A total of 238 radiocarbon datings from materials collected using different sampling procedures was considered for this work. The sedimentation rates varied from less than 2 to $68 \mathrm{~cm} . \mathrm{kyr}^{-1}$. The highest sedimentation rate values were found in a low-energy (ría type) coastal system as well as in the upwelling zones of Santa Catarina and Cabo Frio. The lowest rates were found on the outer shelf and upper slopes. Our results confirm the strong dependency of the shelf currents, with an emphasis to the terrigenous input from the Río de La Plata outflow which is transported via the Brazilian Coastal Current, as well as of the coupled Brazil Current - Intermediate Western Boundary Current (BC-IWBC) dynamics on the sedimentary processes. At least three indicators of the paleo sea level were found at $12200 \mathrm{yr}$ BP (conventional radiocarbon age) (103 meters below sea level - mbsl), 8300-8800 cal yr BP (13 mbsl) and 7700-8100 cal yr BP (6 mbsl).
\end{abstract}

Key words: continental margin, quaternary, radiocarbon, sea-level, sedimentation.

\section{INTRODUCTION}

The northernmost part of the southern Brazilian margin is known as the São Paulo Bight, an arc-shaped embayment extending from $23^{\circ} \mathrm{S}$ to $28^{\circ} \mathrm{S}$ (Zembruscki 1979). Due to the absence of important adjacent fluvial sources, Late Quaternary depositional processes on the São Paulo Bight have been considered for decades to be a result of the reworking of sediments that had been previously deposited at sea level lowstands during the Late Pleistocene. More recently, a series of papers has reevaluated the sedimentary processes on the continental shelf and upper slope in terms of hydrodynamic factors and the input of terrigenous sediments. The latter is especially related to the transport of the Río de La Plata sediments to the Brazilian margin (Mahiques et al. 2004, 2008, Campos et al. 2008).

Correspondence to: Michel Michaelovitch de Mahiques

E-mail:mahiques@usp.br
The hydrodynamic control, together with the relative tectonic stability and the absence of post-glacial rebound, makes the area a favorable site for investigations of the Late Quaternary climatic changes of the southwestern Atlantic. The area has a big potential for studying changes related to the Last Climatic Cycle, sub-Milankovitch variations such as latitudinal shifts of the Intertropical Convergence Zone (ITCZ) (Haug et al. 2001), and variations in the El Niño Southern Oscillation (ENSO) (Woodroffe et al. 2003).

On the other hand, due to flourishing oil and gas exploration activities and related engineering and environmental aspects such as the installation of pipelines and platforms, studies on sedimentary processes are of great importance.

In the last decade, a set of more than two hundred radiocarbon datings have been obtained by the authors using sediment samples from the São Paulo Bight. Most 
of these datings has never been published, although some are available in the scientific literature (Mahiques et al. 2002, 2005, 2007, 2009, Nagai et al. 2009).

Here we present an inventory of the data and analyze the radiocarbon datings in terms of Late Quaternary sedimentary processes and sedimentation rates. In addition, these data provide indications of sea level stabilization periods that occurred prior to the Holocene Climatic Optimum, a subject that is not well understood in this area of study.

Different aspects have been described by other authors that could make it difficult or even impossible to utilize radiocarbon datings in studies of sedimentary and stratigraphical processes in the São Paulo Bight. In particular, inconsistencies between radiocarbon datings and other geological or geochronological indicators may affect the usefulness of this method for examining such processes.

Carroll et al. (2003) analyzed the radiocarbon ages of brachiopod shells found in the first $10 \mathrm{~cm}$ of sediment in cores collected from the inner shelf (between 5 and 23 meters). The authors identified a wide range of ages among these samples, varying from 540-410 to 2420-2240 cal yr BP. In another study comparing radiocarbon datings with ${ }^{210} \mathrm{~Pb}$ and ${ }^{137} \mathrm{Cs}$ data, Figueira et al. (2007) identified a difference of one order of magnitude in estimates of the sedimentation rates in sediment samples from the continental shelf of the area. Finally, Angulo et al. (2008) observed the occurrence of radiocarbon age inversions in the coastal plain adjacent to the study area. The authors therefore defined two sets of radiocarbon ages, e.g., those representing in situ or poorly transported samples, and those indicating allochthonous, highly transported materials.

\section{STUDY AREA}

Figure 1 presents the study area. The ocean floor of the São Paulo Bight shows a rather complex morphology involving channels, canyons, and considerable variations in slope morphology (Furtado et al. 1996). The shelf break is located at a water depth of approximately 140 meters, with the upper slope showing an average gradient of approximately 1:55.

The distribution of surface sediments on the southeastern Brazilian margin was extensively studied in the decade of 1970 and is described in the papers of Rocha et al. (1975) and Kowsmann and Costa (1979). In general, the present sea floor is covered by very fine siliciclastic sands and silts with variable amounts of clay and calcium carbonate. Coarser terrigenous sediments, carbonate gravel and boulder facies found on the outer shelf represent less than $5 \%$ of the present bottom and are generally related to relict sediments that are deposited under lower sea level conditions.

Sedimentary processes in the area have been reevaluated in recent papers in terms of the controlling hydrodynamic processes. On the inner shelf, the sedimentation is mainly determined by the displacement of the Brazilian Coastal Current (Souza and Robinson 2004), which carries sediments from the Río de La Plata and, to a lesser extent, from the southern Brazilian coastal lagoons (Campos et al. 2008, Möller Jr. et al. 2008, Mahiques et al. 2008). On the middle and outer shelves, as well as on the upper slope, the sedimentary processes are mainly influenced by the southward flow of the Brazil Current (BC) along the western Atlantic continental margin (Mahiques et al. 2002, 2004).

\section{METHODS}

In this study, we organized all of the information obtained by the authors on radiocarbon datings performed on sediment samples collected along the São Paulo Bight (Fig. 1). A total of 238 radiocarbon datings obtained from materials collected with different sampling procedures (scuba diving, box cores, piston cores, vibracores) (see Tables I to IV for details) were considered for this work.

With few exceptions, the content of suitable carbonate materials in the collected cores was insufficient for radiocarbon dating, and at several sampling sites the bulk organic fraction was used for dating. Almost all of the samples were AMS dated at Beta Analytic Inc. (Miami, USA). Calibrated ages of marine samples (preserved bivalve shells, corals, specimens of the planktonic foraminifer Globigerinoides ruber on core 7485 and the benthic foraminifer Elphydium sp on core SSB01) were calculated using Calib software, version 5.0.2html, available at http://calib.qub.ac.uk/calib/. We employed the standard marine correction of 408 years and a regional reservoir effect of $\Delta \mathrm{R}=12 \pm 60$, 


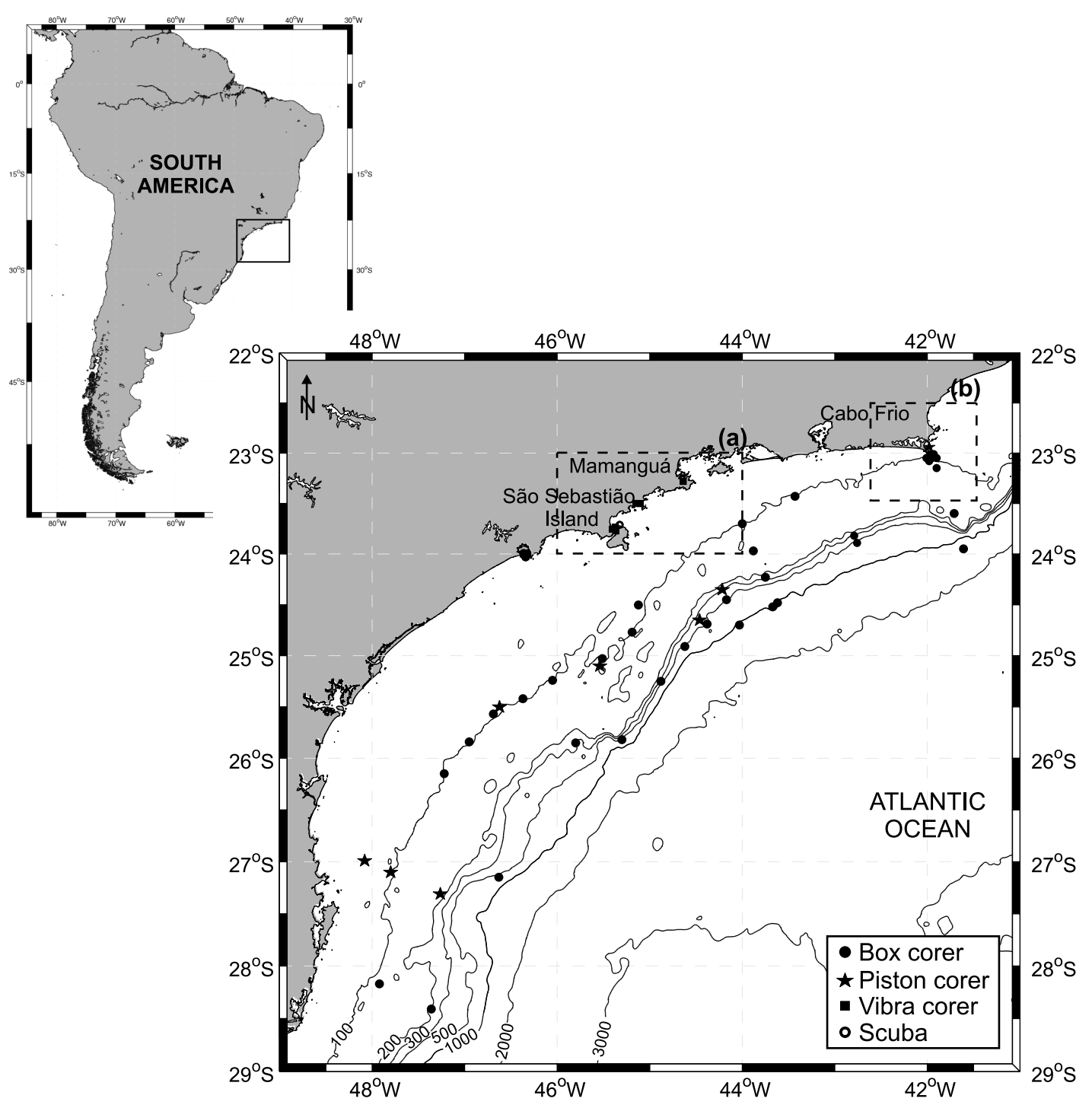

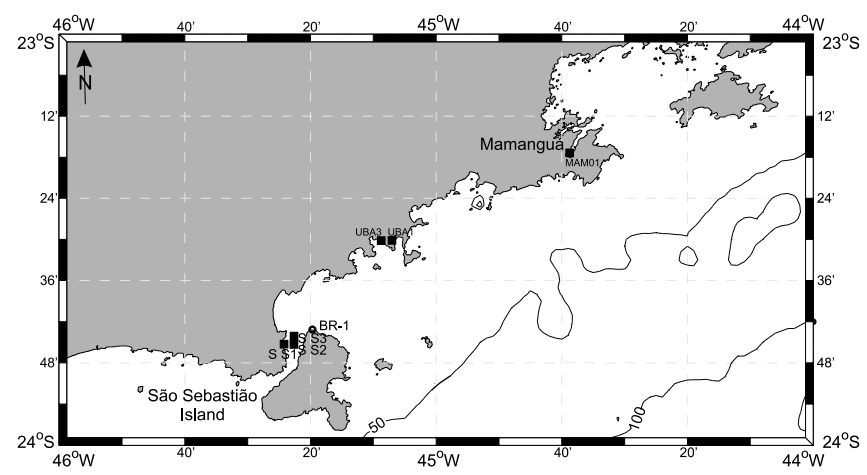

(a)

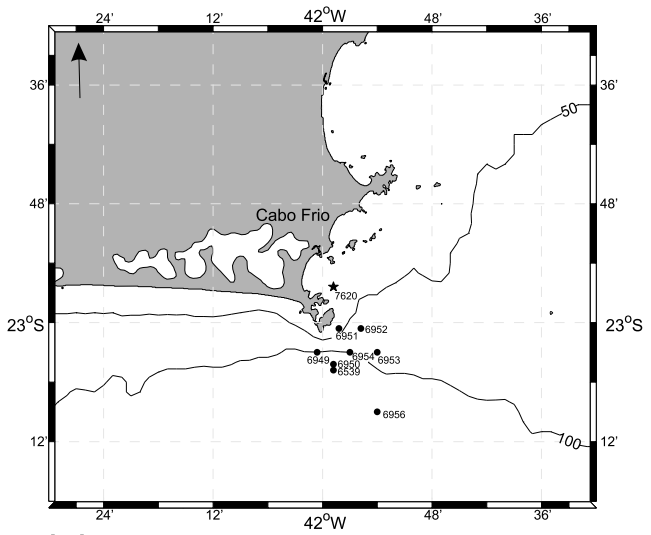

(b)

Fig. 1 - Location of the study area and samples considered in this study. 
corresponding to the average value of eleven samples reported for the SE Brazilian coast between the latitudes $23^{\circ} \mathrm{S}$ and $28^{\circ} \mathrm{S}$ (Nadal de Masi 1999, Eastoe et al. 2002, Angulo et al. 2005) and the Marine04 Calibration Dataset (Hughen et al. 2004). For terrestrial material, the Southern Hemisphere Calibration Curve (McCormac et al. 2004) was used. Finally, for organic matter samples, the Mixed-Marine calibration curve was used. Estimates of marine versus terrestrial percentages are based on $\delta^{13} \mathrm{C}$ end-members for the São Paulo Bight $(-19.00 \%$ PDB for marine and $-26.00 \%$ PDB $)$ (Mahiques et al. 1999).

Average sedimentation rates were obtained by linear interpolation of calibrated ages only for those samples that presented at least three datings with Holocene ages, assuming that the surface sediment was modern $(0 \mathrm{kyr})$. For each of these average rates, the significance level was determined as shown in Tables I to III.

\section{RESULTS}

Tables I to III present the results of the radiocarbon datings. In order to develop a better comprehension of the sedimentary processes in the context of these data, the samples were divided into three different geomorphological sectors, i.e., "Coast", comprising the submerged coastal environments up to the 20-meter isobath; "Inner and Middle Shelf", comprising the samples located from the 20-meter to the 100-meter isobath; and "Outer Shelf/Upper Slope”, beyond the 100-meter isobath.

The samples from the Coast sector comprise 69 datings that were performed on seven vibracores, five box cores and four beach rock samples collected by scuba diving (Table I). In this sector, most of the datings showed Mid- to Late Holocene ages with the exception of the base of one core (SS1), which showed a coherent sequence of datings (foraminifers and organic matter) with conventional radiocarbon ages ranging from ca. $39,300 \mathrm{yr}$ BP to $10,240 \mathrm{yr}$ BP. Age inversions are not rare in core samples from this sector, and incoherencies between carbonate-based (mollusk) and organic matter-based radiocarbon ages were also observed. Due to these complications, the estimates of sedimentation rates were performed at only three vibracore and two box core stations, providing a range varying from $12 \pm 2 \mathrm{~cm} \cdot \mathrm{kyr}^{-1}$ to $68 \pm 2 \mathrm{~cm} \cdot \mathrm{kyr}^{-1}$. The highest sedimentation rate value was obtained from a vibracore sampled in the Mamanguá inlet $\left(23^{\circ} 17.40^{\prime} \mathrm{S}\right.$ $044^{\circ} 38.88^{\prime} \mathrm{W}$ ), a coastal system with geomorphological and sedimentological characteristics that are very similar to those present in the northwestern Iberian Rías (Méndez and Vilas 2005). The lowest sedimentation rate values were observed in cores collected in the São Sebastião Channel $\left(23^{\circ} 45.12^{\prime} \mathrm{S}-045^{\circ} 22.62^{\prime} \mathrm{W}\right)$ and in Santos Bay $\left(24^{\circ} 00.00^{\prime} \mathrm{S}-046^{\circ} 20.58^{\prime} \mathrm{W}\right)$, two coastal systems in which the wave action is more effective.

Samples from the Inner and Middle Shelf sector (Table II) correspond to 98 datings from 17 box cores and 5 piston cores. Most of these show a rather coherent pattern of radiocarbon dating, with only a few age inversions at the base of some cores and a prevalence of Mid- to Late-Holocene calibrated ages. One of the piston core samples $\left(7616,25^{\circ} 05.88^{\prime} \mathrm{S}-045^{\circ} 38.64^{\prime} \mathrm{W}\right)$ presented an erosional contact at its lowermost levels. Corresponding radiocarbon hiatuses detected at these levels showed indications of the transition from Late Pleistocene sandy beach facies, with conventional radiocarbon ages ranging from $13370 \pm 70$ to $12170 \pm 70 \mathrm{yr}$ BP, to a Mid-Holocene muddy shelf sediment.

Sedimentation rate estimates were calculated based on 11 box cores and 5 piston cores; the highest values $\left(40 \pm 9\right.$ and $\left.62 \pm 10 \mathrm{~cm} \cdot \mathrm{kyr}^{-1}\right)$ were found in samples from the Cabo Frio and the Santa Catarina upwelling zones. Lower sedimentation rate values (lower than $15 \mathrm{~cm} \cdot \mathrm{kyr}^{-1}$ ) were found in two samples located close to the 100-meter isobath (samples 6561 and 7605).

Samples from the Outer Shelf/Upper Slope sector (Table III) comprise 71 datings from 21 box cores and 3 piston cores. This sector is characterized by very low sedimentation rate values that were present in several samples. These values indicate the presence of relict terms such as those found in samples 6573, 6626, 6652 and 6680, in which Pleistocene ages were reached at a thickness of few centimeters of sediment (see Table III for location). Also, a 4.5-meter long core (7607, $27^{\circ} 22.14^{\prime} \mathrm{S}-47^{\circ} 08.40^{\prime} \mathrm{W}$ ), which was collected at 287 meters below sea level (mbsl) and was composed exclusively of massive mud, exhibited a sedimentary column with a completely fuzzy pattern and ages ranging between 30000 and $40000 \mathrm{yr}$ BP These features lead us to consider this latter core as an evidence of a relict 


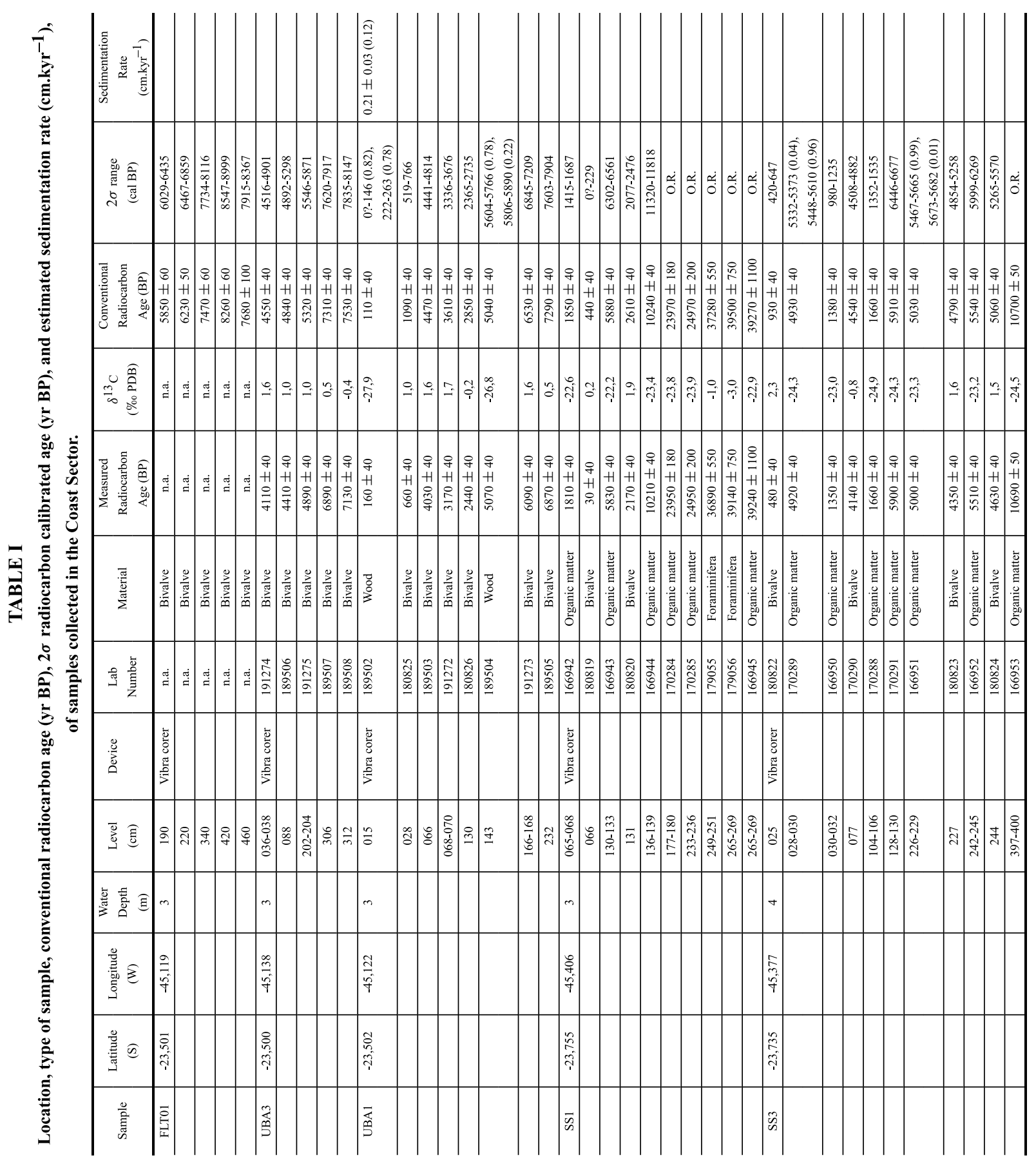




\begin{tabular}{|c|c|c|c|c|c|c|c|c|c|c|c|c|c|c|c|c|c|c|c|c|c|c|c|c|}
\hline 总 & 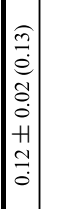 & & & & & 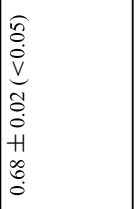 & & & & & & & & 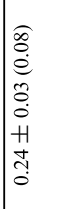 & & 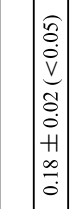 & & & & & & & & \\
\hline 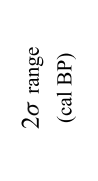 & 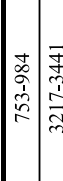 & 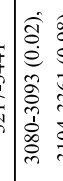 & 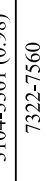 & 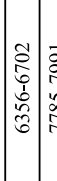 & $\frac{1}{0}$ & 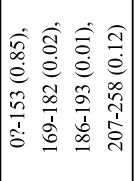 & 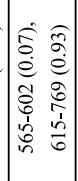 & 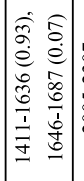 & & 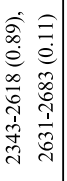 & 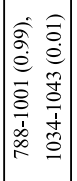 & 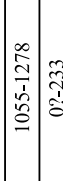 & 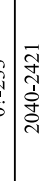 & 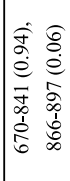 & 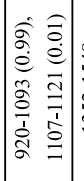 & 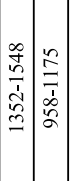 & 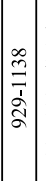 & 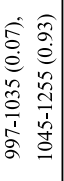 & 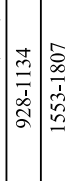 & \begin{tabular}{|c|c|} 
\\
0 \\
0 \\
0 \\
0 \\
0 \\
$\infty$ \\
$\infty$
\end{tabular} & 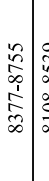 & & 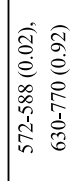 & 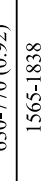 \\
\hline 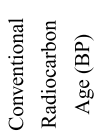 & 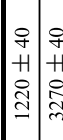 & 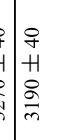 & $\begin{array}{l}0 \\
0 \\
1 \\
+1 \\
0 \\
0 \\
0 \\
0\end{array}$ & 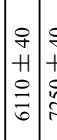 & 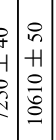 & 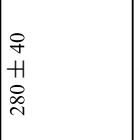 & 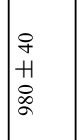 & $\mid \begin{array}{l}q \\
+ \\
+ \\
\infty \\
\infty \\
\infty \\
\end{array}$ & 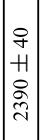 & 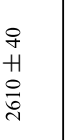 & $\begin{array}{l}\text { o } \\
\text { o } \\
1 \\
0 \\
9 \\
\end{array}$ & 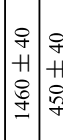 & 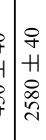 & 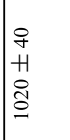 & 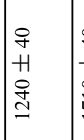 & 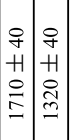 & 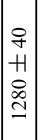 & 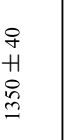 & 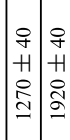 & $\mid \begin{array}{c}\mid \\
0 \\
+ \\
+1 \\
0 \\
2 \\
2 \\
1\end{array}$ & 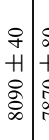 & $\begin{array}{l}0 \\
0 \\
0 \\
0 \\
0 \\
0 \\
0\end{array}$ & 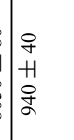 & $\begin{array}{l}\text { o } \\
+ \\
\text { H } \\
\text { d } \\
\end{array}$ \\
\hline 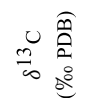 & $\overrightarrow{\vec{\imath}}$ & רִ & $\overrightarrow{\vec{T}}$ & $\therefore=$ & 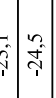 & 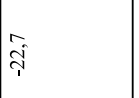 & $\overrightarrow{\text { जิ }}$ & ఫે & $\left|\begin{array}{l}\infty \\
\overrightarrow{\vec{T}}\end{array}\right|$ & iิ & 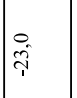 & $\mid \frac{1}{4}$ & iे & $\underset{\gamma}{\tilde{z}}$ & ๙े. & \begin{tabular}{l|l}
0 \\
\hdashline \\
\hdashline
\end{tabular} & خิ & ते & 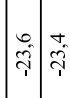 & $\because$ & : & 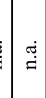 & $\vec{\tau}$ & $\overrightarrow{\hat{N}}$ \\
\hline 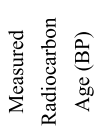 & 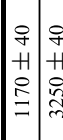 & 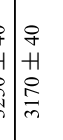 & $\begin{array}{l}0 \\
n \\
+1 \\
0 \\
0 \\
6 \\
6\end{array}$ & 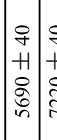 & 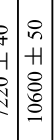 & $\mid$ & $\begin{array}{l}q \\
+ \\
+1 \\
0 \\
0\end{array}$ & 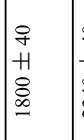 & 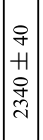 & 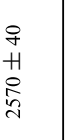 & 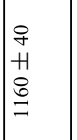 & 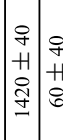 & 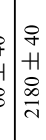 & $\mid$\begin{tabular}{|l}
9 \\
+ \\
0 \\
2 \\
2
\end{tabular} & $\mid$ & 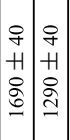 & 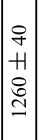 & 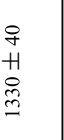 & 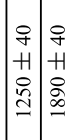 & 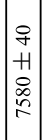 & \begin{tabular}{l|l} 
& \\
+ \\
+ \\
1 \\
$\vdots$ \\
$\vdots$ \\
$\infty$
\end{tabular} & $\stackrel{g}{g}$ & $\begin{array}{l}8 \\
+ \\
+1 \\
0 \\
a\end{array}$ & 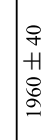 \\
\hline 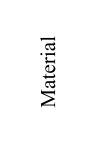 & 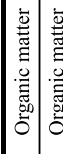 & 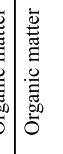 & 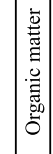 & 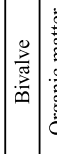 & 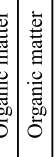 & 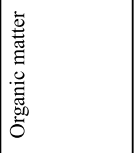 & 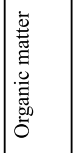 & 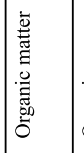 & 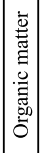 & 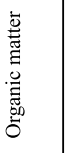 & 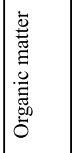 & 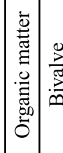 & 跣 & 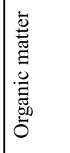 & 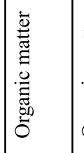 & 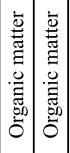 & 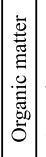 & 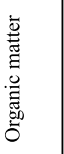 & 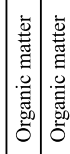 & 辛 & 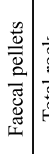 & 豙 & 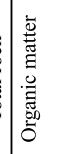 & 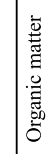 \\
\hline 祃旁 & 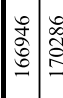 & \begin{tabular}{c} 
: \\
$\vdots$ \\
\hdashline
\end{tabular} & $\mid$ & 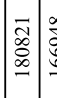 & 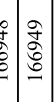 & 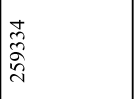 & 染 & 总 & & 㐫 & 总 & 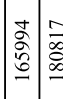 & $=\left[\begin{array}{l}\infty \\
0 \\
0 \\
0 \\
0 \\
0 \\
0\end{array}\right.$ & 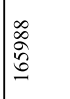 & $\mid$ & $\begin{array}{lll}2 & \\
0 \\
0 \\
0 \\
0\end{array}$ & 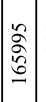 & 离 & 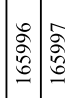 & 景 & 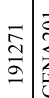 & & 辰 & 足 \\
\hline 总 & 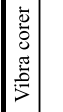 & & & & & 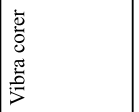 & & & & & 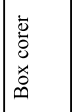 & 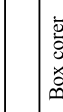 & & 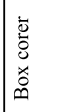 & & 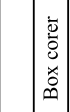 & & & & 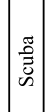 & & & 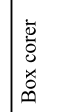 & \\
\hline 总 & 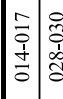 & 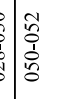 & 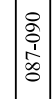 & $\infty \begin{array}{l}\infty \\
\varnothing \\
\varnothing\end{array}$ & 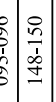 & $\delta$ & 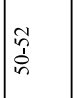 & $\frac{\delta}{\dot{d}}$ & 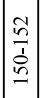 & 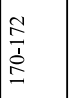 & 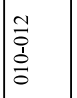 & 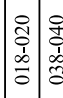 & \begin{tabular}{c|c}
0 \\
$\vdots$ \\
$\vdots$ \\
$\vdots$ \\
$\vdots$
\end{tabular} & $\begin{array}{l}\frac{1}{0} \\
\frac{0}{0} \\
\frac{1}{0}\end{array}$ & 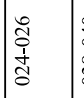 & 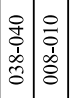 & $\left|\begin{array}{l}\frac{\partial}{0} \\
\dot{\partial} \\
\frac{\partial}{\partial}\end{array}\right|$ & त్రี & 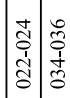 & 8 & ¿ & 8 & 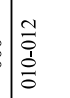 & 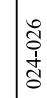 \\
\hline 总言 & in & & & & & o & & & & & a & 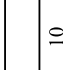 & & $\cong$ & & $=$ & & & & $\approx$ & & & \pm & \pm \\
\hline 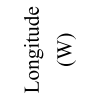 & 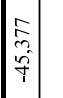 & & & & & $\begin{array}{l}\text { 悉 } \\
\text { 竎 }\end{array}$ & & & & & 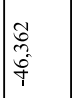 & 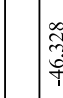 & & 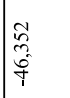 & & 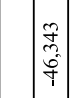 & & & & 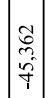 & & & 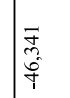 & 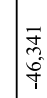 \\
\hline 害 & $\mid \begin{array}{c}\hat{2} \\
\hat{\imath} \\
\hat{i}\end{array}$ & & & & & $\begin{array}{l}\text { के } \\
\text { ते } \\
\text { ते }\end{array}$ & & & & & 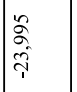 & 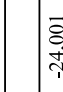 & & 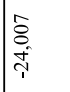 & & $\mid$ & & & & 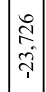 & & & 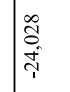 & \begin{tabular}{l}
$\infty$ \\
$\widetilde{8}$ \\
\multirow{7}{*}{+0}
\end{tabular} \\
\hline $\begin{array}{l}\frac{2}{0} \\
\frac{1}{y_{0}}\end{array}$ & $\tilde{\omega}$ & & & & & $\sum_{\substack{\infty \\
\Sigma}}^{\overline{0}}$ & & & & & f & Z & & 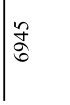 & & $\mid \begin{array}{l}0 \\
0 \\
0 \\
0\end{array}$ & & & & T. & $\approx$ & & 产 & 웡 \\
\hline
\end{tabular}




\begin{tabular}{|c|c|c|c|c|c|c|c|c|c|c|c|c|c|c|c|c|c|c|c|c|c|c|c|c|c|c|}
\hline$\hat{\tilde{T}}$ & 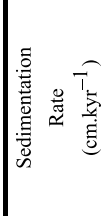 & & & & & & & & & & 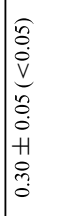 & & & 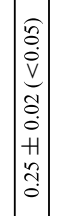 & & & 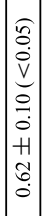 & & & & & & & & & \\
\hline : & 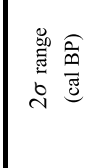 & $\mid \begin{array}{l}n \\
\vdots \\
0 \\
0 \\
\vdots \\
n\end{array}$ & 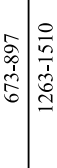 & 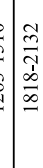 & 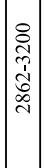 & 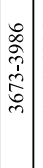 & 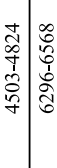 & 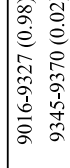 & & 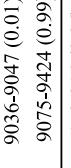 & 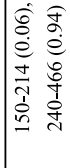 & 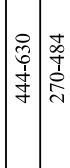 & 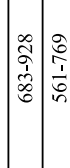 & 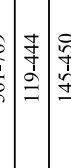 & 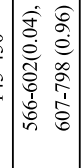 & & $\frac{\infty}{i}$ & 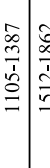 & $\mid$ & & 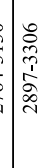 & 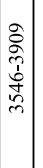 & & 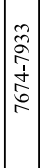 & & \\
\hline 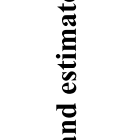 & 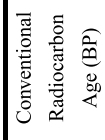 & $\begin{array}{l}0 \\
0 \\
+ \\
+1 \\
0 \\
\infty \\
\infty\end{array}$ & 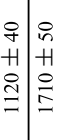 & 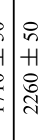 & 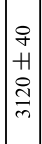 & $\begin{array}{ll} & \\
q \\
+ \\
+ \\
0 \\
\infty \\
m\end{array}$ & 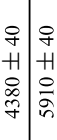 & $\mid$ & 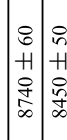 & 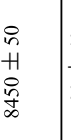 & \begin{tabular}{|l} 
\\
+ \\
+1 \\
8 \\
0 \\
$i n$
\end{tabular} & \begin{tabular}{|l|l} 
& \\
9 & 8 \\
+1 & +1 \\
0 & 0 \\
0 & 0 \\
\end{tabular} & 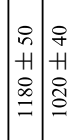 & 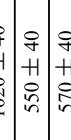 & 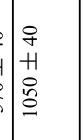 & 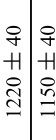 & 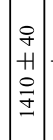 & 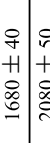 & $\mid \begin{array}{l}0 \\
0 \\
+1 \\
0 \\
0 \\
a \\
\end{array}$ & & 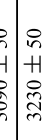 & $\begin{array}{ll} & \\
o \\
+ \\
+ \\
⿱ \\
0 \\
m\end{array}$ & & \begin{tabular}{|l|}
0 \\
9 \\
+1 \\
0 \\
$\vdots$ \\
0 \\
1
\end{tabular} & & 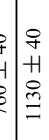 \\
\hline 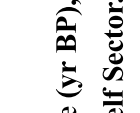 & 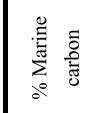 & b & : & 5 & I & t & $\stackrel{t}{0}$ & in & & f & I & $\Rightarrow \quad I$ & $: 8$ & $8 \pi$ & $F$ & $\pm \div$ & $\vec{\sigma}$ & $\grave{\infty} \otimes$ & $\bar{\infty}$ & & $=\infty$ & $\vec{\sigma}$ & $\infty_{\infty}^{\infty}$ & 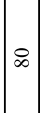 & $\therefore \approx$ & $\approx \approx$ \\
\hline 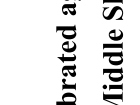 & 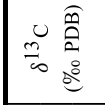 & $\overrightarrow{\hat{i}}$ & $\begin{array}{ll}\vec{N} \\
\vec{T}\end{array}$ & 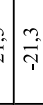 & $\mid \begin{array}{l}\mid \\
\vec{T}\end{array}$ & | & $\overrightarrow{\vec{T}}$ & $\overrightarrow{\vec{T}}$ & & 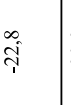 & 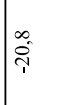 & 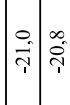 & $\overrightarrow{\vec{T}}=\vec{T}$ & 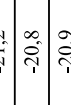 & $\mid$ & & $\mid$\begin{tabular}{l}
0 \\
\hdashline \\
\hdashline
\end{tabular} & \begin{tabular}{l|l}
$\infty$ & $\infty$ \\
\hdashline & 0 \\
\hdashline & 0
\end{tabular} & 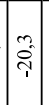 & & î̀ & 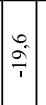 & & : & & خิ \\
\hline 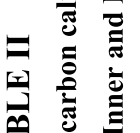 & 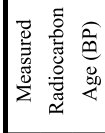 & $\mid$\begin{tabular}{l}
0 \\
8 \\
+ \\
\hdashline \\
\hdashline \\
\hdashline
\end{tabular} & 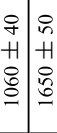 & 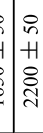 & \begin{tabular}{|c|} 
\\
$q$ \\
+1 \\
0 \\
0 \\
0 \\
\end{tabular} & 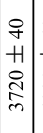 & 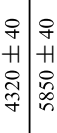 & 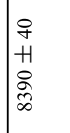 & 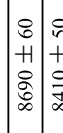 & $\begin{array}{l}8 \\
b \\
+1 \\
\frac{0}{+} \\
\infty\end{array}$ & $\begin{array}{l}\text { o } \\
+ \\
+1 \\
\text { î } \\
\text { in }\end{array}$ & 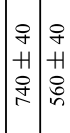 & 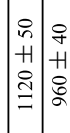 & 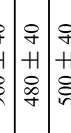 & 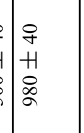 & 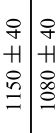 & 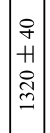 & 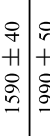 & 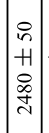 & & 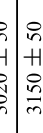 & 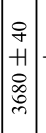 & 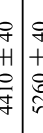 & $\mid \begin{array}{l}\mid \\
8 \\
+1 \\
0 \\
\frac{9}{2} \\
\end{array}$ & 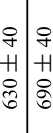 & 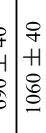 \\
\hline 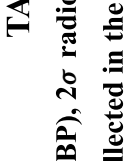 & 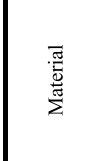 & 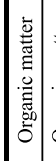 & 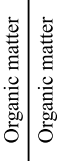 & 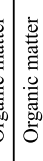 & & 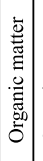 & 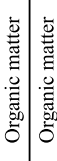 & 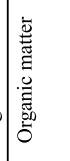 & 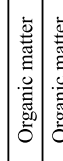 & 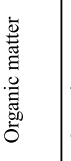 & 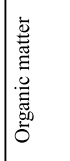 & 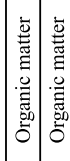 & 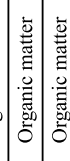 & 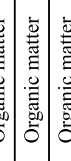 & 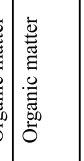 & 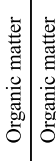 & 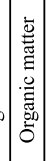 & 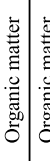 & 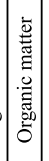 & 离 & 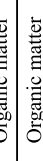 & 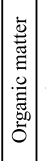 & 5 & 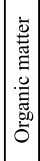 & 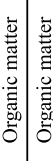 & 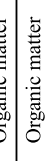 \\
\hline 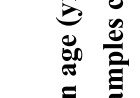 & 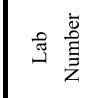 & 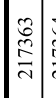 & 壱旅 & 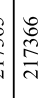 & 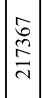 & $\mid$ & 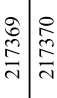 & 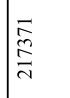 & & 商 & 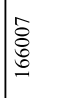 & 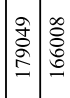 & 总 & 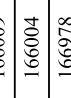 & 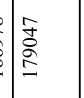 & 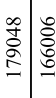 & जे & 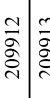 & $\mid$ & & 票 & $\mid$ & & & 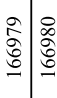 & : \\
\hline 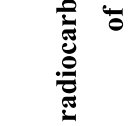 & 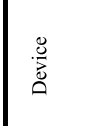 & 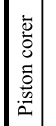 & & & & & & & & & 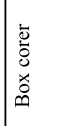 & & & 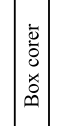 & & & 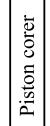 & & & & & & & & & \\
\hline 节 & 兽 & $\left|\begin{array}{c}0 \\
\vdots \\
\vdots \\
\vdots\end{array}\right|$ & 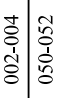 & & 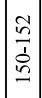 & : & 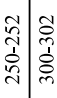 & 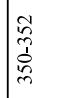 & 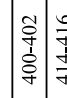 & 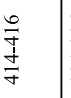 & 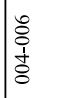 & 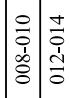 & 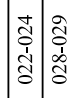 & 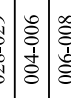 & $\begin{array}{l}\frac{\pi}{\partial} \\
\frac{\partial}{\partial}\end{array}$ & 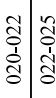 & \begin{tabular}{|c|c}
0 \\
$\vdots$ \\
$\vdots$ \\
$\vdots$ \\
$\vdots$ \\
\end{tabular} & 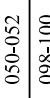 & 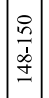 & & 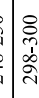 & $\mid$ & 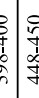 & & & 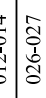 \\
\hline$\cong$ & 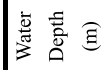 & F & & & & & & & & & $\stackrel{\infty}{n}$ & & & 8 & & & 8 & & & & & & & & m & \\
\hline$\stackrel{0}{\circ}$ & 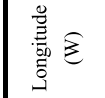 & 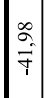 & & & & & & & & & 17 & & & Fे & & & $\left|\begin{array}{c}\infty \\
0 \\
0 \\
f \\
f\end{array}\right|$ & & & & & & & & : & \\
\hline . & 善 & 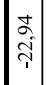 & & & & & & & & & तु & & & כ & & & 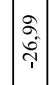 & & & & & & & & . & \\
\hline & 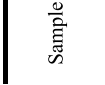 & $\mid$ & & & & & & & & & 80 & & & 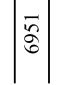 & & & |cto & & & & & & & & ถิ & \\
\hline
\end{tabular}




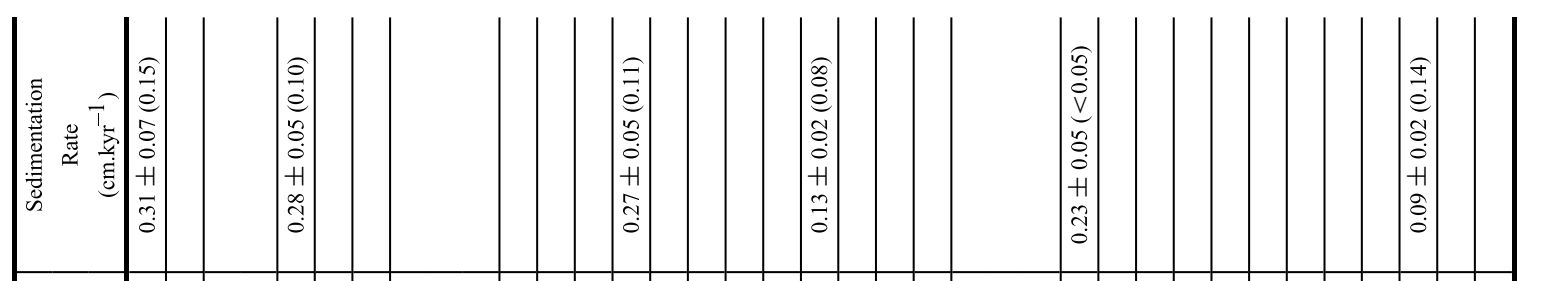

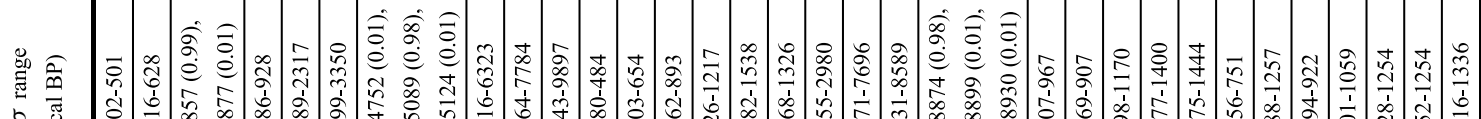

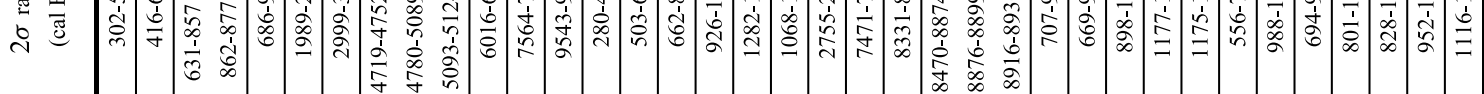

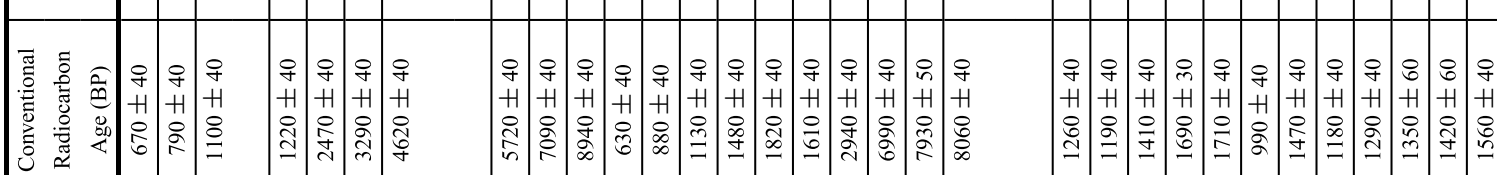

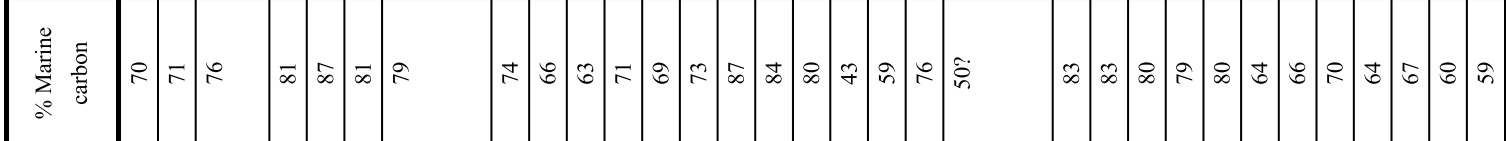

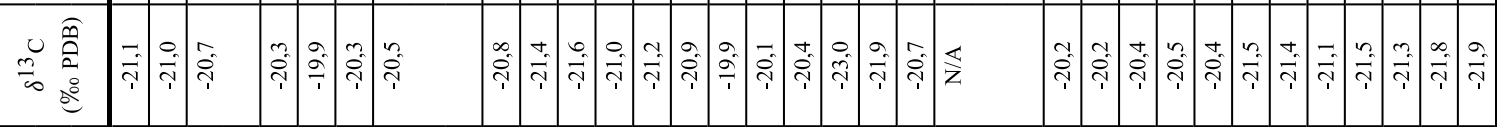

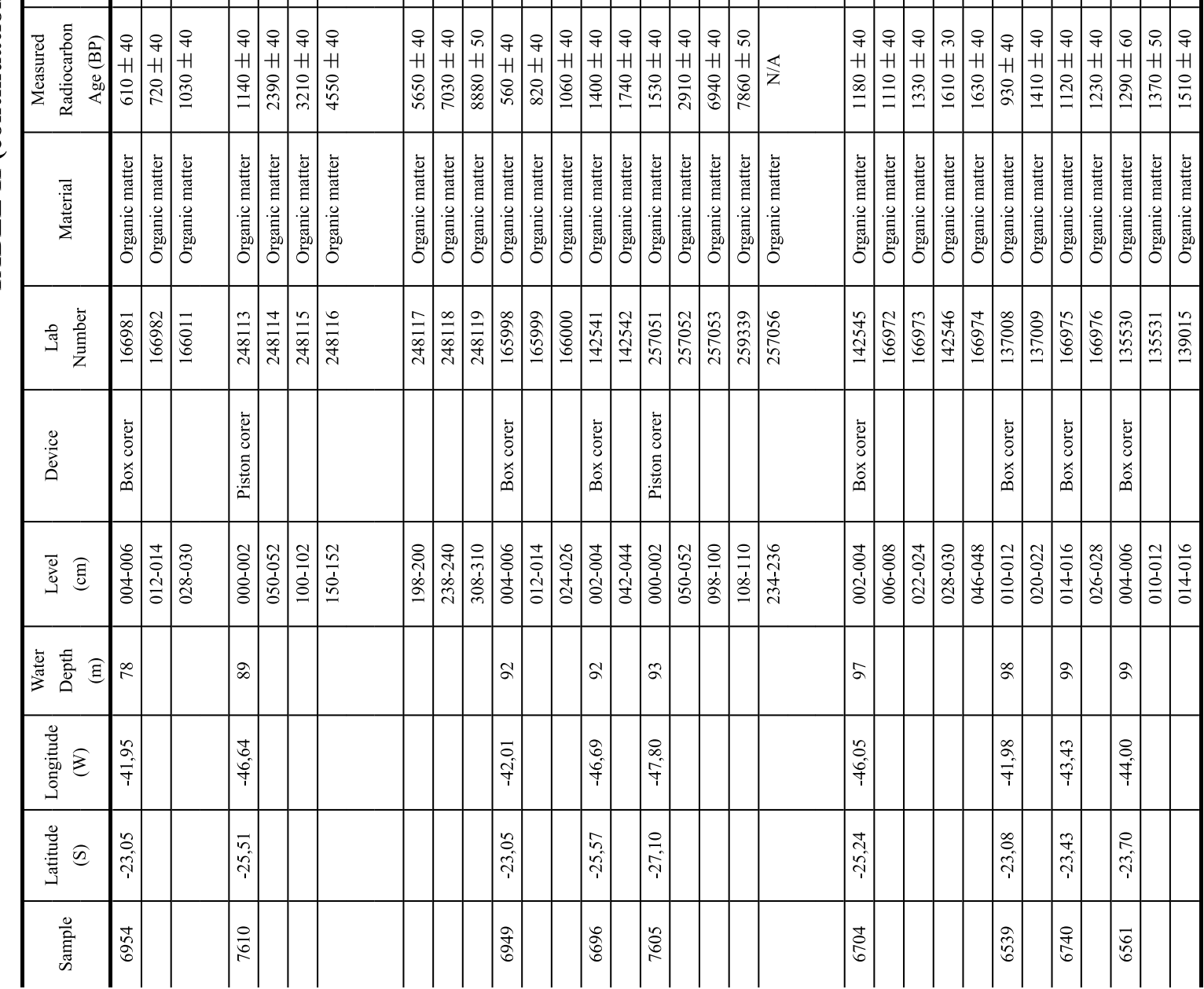



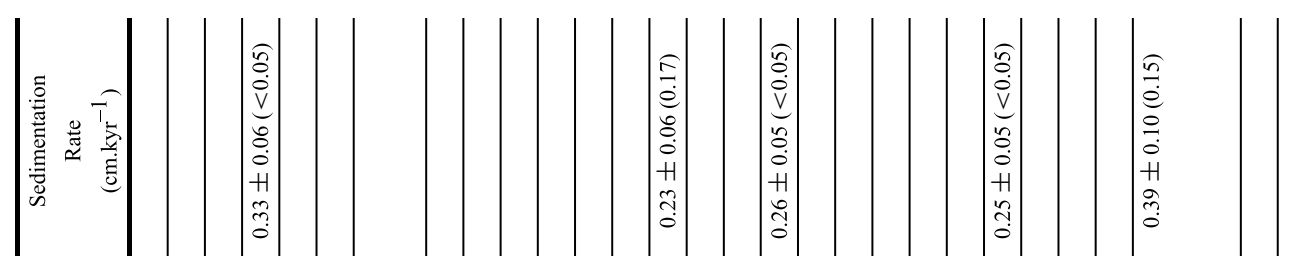

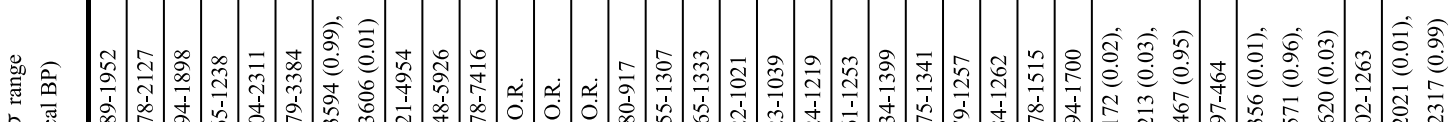

完 至

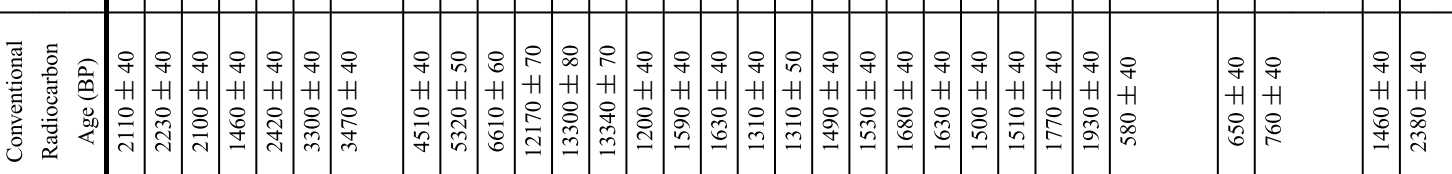

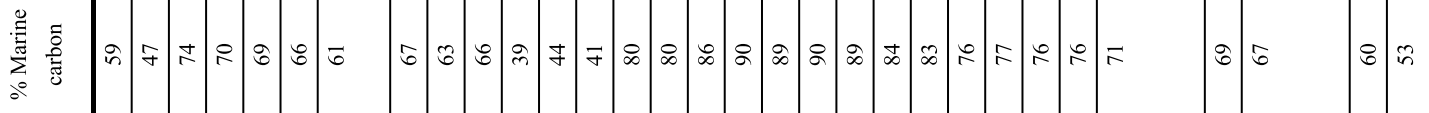

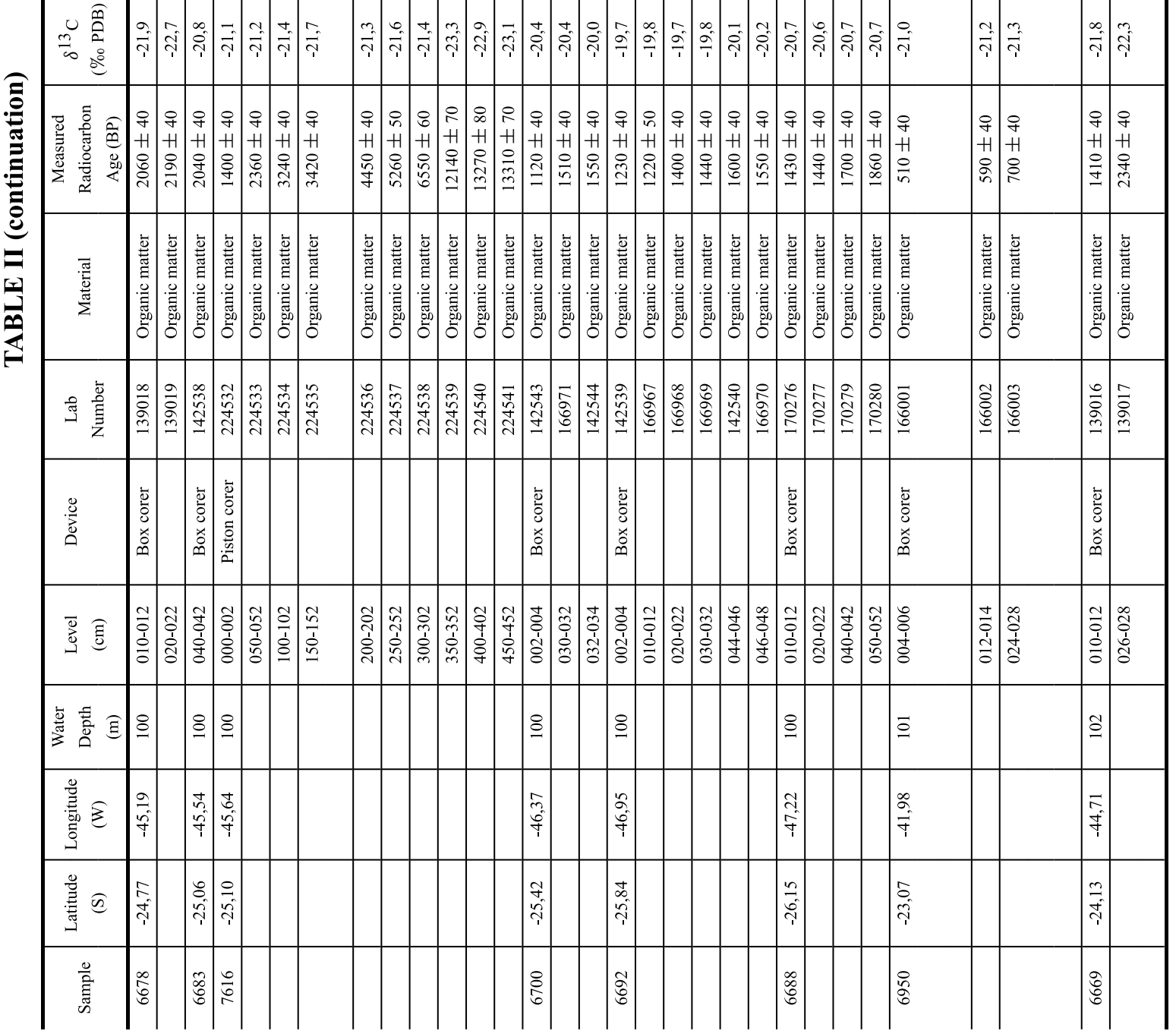




\begin{tabular}{|c|c|c|c|c|c|c|c|c|c|c|c|c|c|c|c|c|c|c|c|c|c|c|c|c|}
\hline$\stackrel{\overbrace{}}{\stackrel{T}{\vec{t}}}$ & 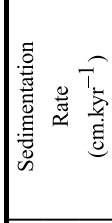 & 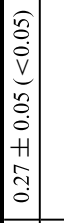 & & & $\mid$\begin{tabular}{l|}
0 \\
0 \\
0 \\
0 \\
0 \\
0 \\
+1 \\
$\infty$ \\
0 \\
0
\end{tabular} & & & & & & & & & & & & & & & & & & & \\
\hline 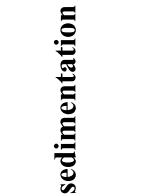 & 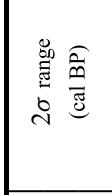 & 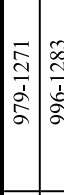 & 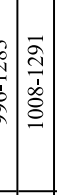 & 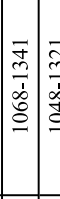 & 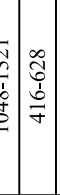 & 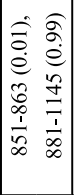 & 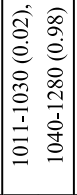 & 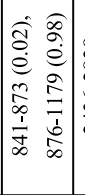 & 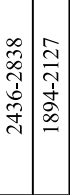 & 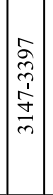 & 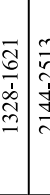 & 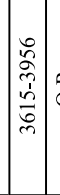 & 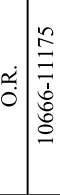 & 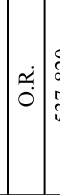 & & 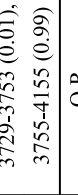 & 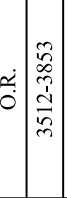 & $\left|\begin{array}{c}0 \\
0 \\
0 \\
0 \\
\vdots \\
\vdots \\
\vdots \\
0 \\
0\end{array}\right|$ & 势 & 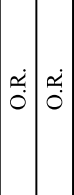 & $\approx$ & & & \\
\hline 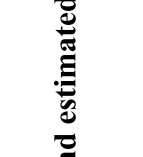 & 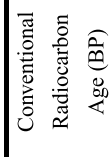 & 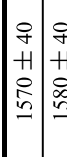 & 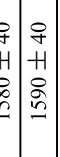 & 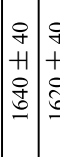 & 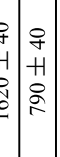 & 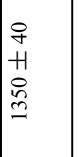 & $\begin{array}{l}9 \\
+ \\
+1 \\
0 \\
\underline{n} \\
\underline{n}\end{array}$ & $\mid \begin{array}{l}8 \\
+1 \\
+1 \\
0 \\
0 \\
9\end{array}$ & 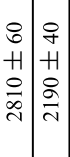 & 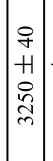 & 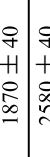 & 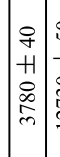 & 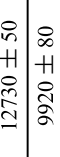 & 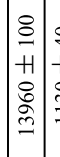 & & & 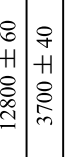 & 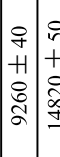 & 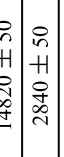 & 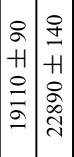 & 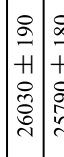 & 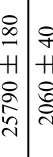 & & \\
\hline 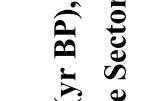 & 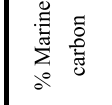 & $2 \sigma$ & 512 & $|\hat{\infty}|$ & $\therefore$ & 8 & 8 & 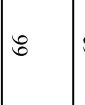 & $8 D$ & $F$ & & 2 & $8=$ & 8 & & 8 & $0 \approx$ & in 8 & 5 & in $\because$ & in & $\stackrel{8}{-}$ & & 8 \\
\hline 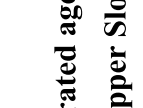 & 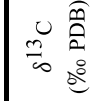 & 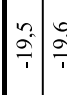 & $\div$ & \begin{tabular}{lll}
2 & $\infty$ \\
\hdashline & 0 & 0
\end{tabular} & $\begin{array}{lll} \\
ن\end{array}$ & $\overrightarrow{\vec{T}}$ & $\vec{y}$ & $\overrightarrow{\vec{T}}$ & 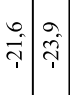 & 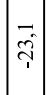 & خे & 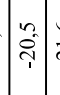 & דَ. & $\vec{\sim}$ & $\hat{i}$ & $\vec{t}$ & خิ & 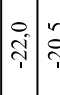 & $\vec{i}$ & $\overrightarrow{\vec{T}} \overrightarrow{\hat{T}}$ & 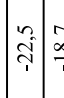 & & & $\frac{7}{\top}$ \\
\hline 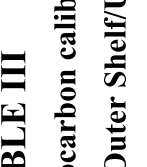 & 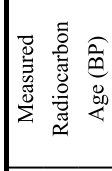 & 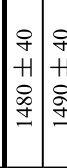 & 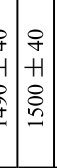 & 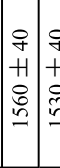 & 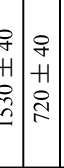 & 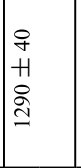 & $\mid \begin{array}{l}9 \\
7 \\
+1 \\
8 \\
9 \\
9\end{array}$ & $\mid \begin{array}{l}8 \\
0 \\
11 \\
8 \\
0 \\
1\end{array}$ & 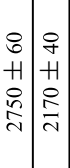 & $\left|\begin{array}{l}q \\
o \\
+1 \\
o \\
m \\
m\end{array}\right|$ & 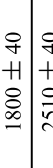 & 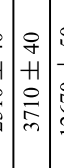 & 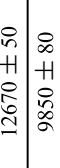 & $\left|\begin{array}{c|c}8 \\
\hdashline \\
+1 \\
0 \\
\vdots \\
0 \\
\hdashline\end{array}\right|$ & & & 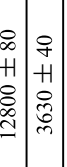 & 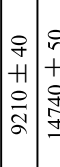 & 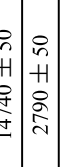 & 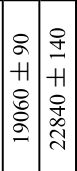 & 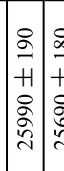 & 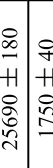 & & $\mid \begin{array}{l}8 \\
1 \\
1 \\
0 \\
0 \\
-3 \\
-3\end{array}$ \\
\hline 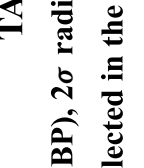 & 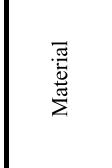 & 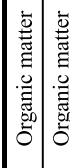 & 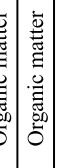 & 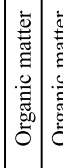 & 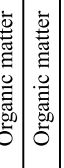 & 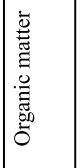 & 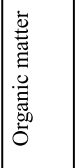 & 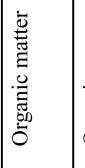 & 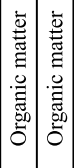 & 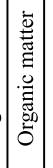 & 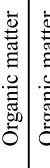 & . & 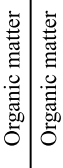 & 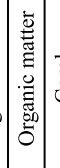 & & & 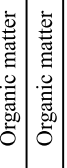 & 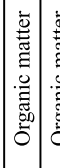 & 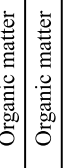 & 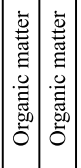 & 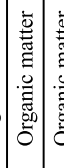 & 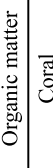 & & 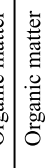 \\
\hline 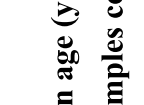 & 导营 & 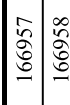 & $\begin{array}{l}0 \\
0 \\
0\end{array}$ & 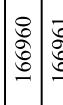 & $\begin{array}{lll}0 & 0 \\
0 & 0 \\
0 & 0 \\
0 & 0 \\
0 & 0\end{array}$ & 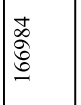 & 畓 & 总 & 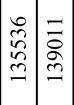 & 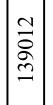 & 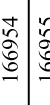 & בิ⿱亠䒑十心 & 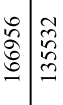 & $\begin{array}{l}\tilde{n} \\
\hat{n} \\
\bumpeq\end{array}$ & 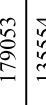 & 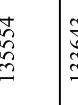 & 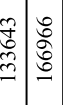 & $\left(\begin{array}{ll}0 \\
0 \\
0 \\
0 \\
\hdashline\end{array}\right.$ & 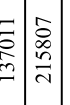 & 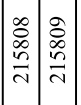 & 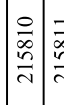 & 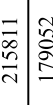 & & 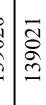 \\
\hline 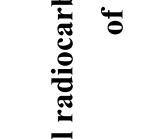 & $\frac{8}{5}$ & 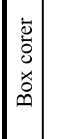 & & & 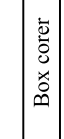 & & & 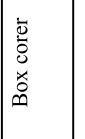 & 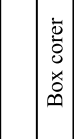 & & 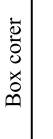 & & 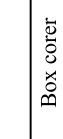 & & & & 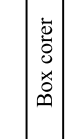 & 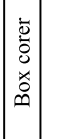 & 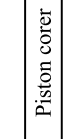 & & & 递 & 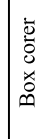 & \\
\hline 苞 & 总 & 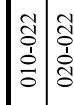 & 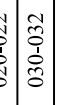 & 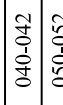 & 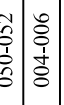 & 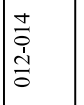 & 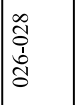 & $\begin{array}{l}0 \\
0 \\
0 \\
\dot{0} \\
\dot{0} \\
0\end{array}$ & 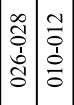 & 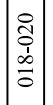 & 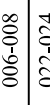 & 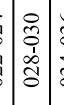 & 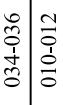 & 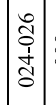 & \& & 豙 & 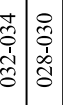 & 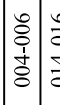 & 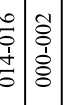 & 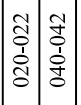 & 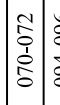 & 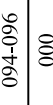 & & 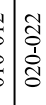 \\
\hline in & 总言 & $\equiv$ & & & $\stackrel{\infty}{=}$ & & & $\stackrel{m}{m}$ & 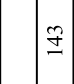 & & ఏ & & $\overrightarrow{\text { ते }}$ & & 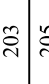 & : & స: & $\vec{\sim}$ & 於 & & & $\tilde{\curvearrowright}$ & సે & \\
\hline 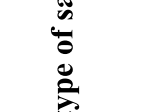 & 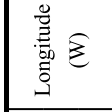 & $\mid \begin{array}{l}\approx \\
\tilde{f} \\
\tilde{f}\end{array}$ & & & $\left|\begin{array}{c}2 \\
\overrightarrow{7} \\
\overrightarrow{7}\end{array}\right|$ & & & 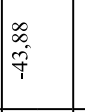 & $\vec{F}$ & & 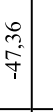 & & 过 & & & ch & $\left|\begin{array}{c}0 \\
0 \\
q \\
q \\
q\end{array}\right|$ & $\mid \begin{array}{l}\hat{\hat{\alpha}} \\
\hat{\gamma}\end{array}$ & $\mid \begin{array}{l}m \\
\dot{y} \\
\dot{f}\end{array}$ & & & $\exists$ & 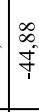 & \\
\hline 䒿 & 善司 & 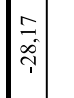 & & & 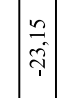 & & & 云 & {[} & & ণ & & 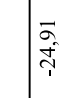 & & ઼ָ & 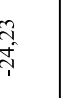 & 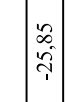 & 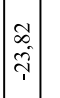 & $\mid$ & & & $\bar{y}$ & & \\
\hline & 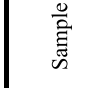 & 勇 & & & 告 & & & స్ర్ర & 1 & & $\bar{\Xi}$ & & 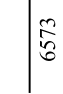 & & & 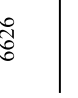 & $\mid$ & $\mid$ & 离 & & & $\stackrel{8}{\circ}$ & : & \\
\hline
\end{tabular}




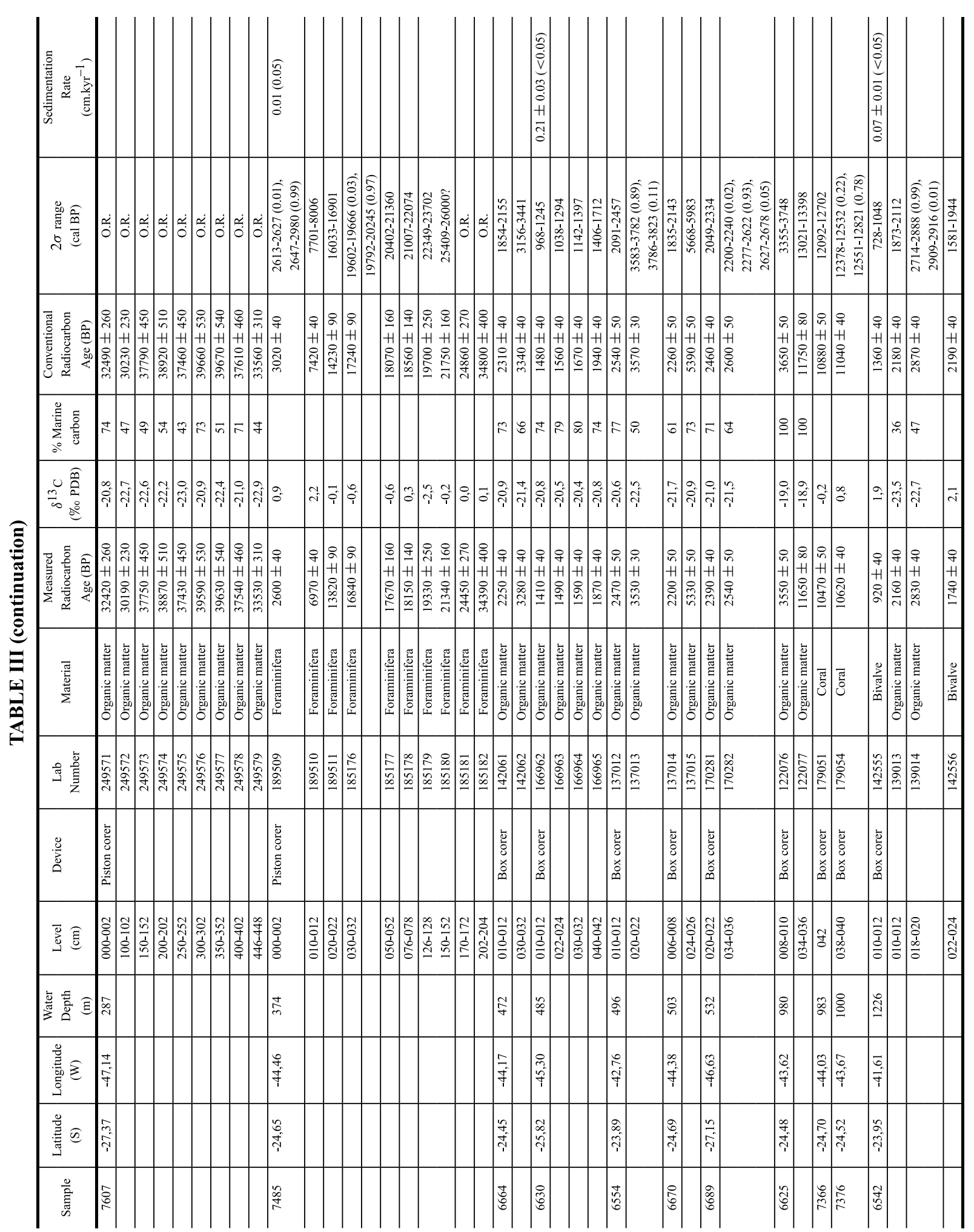


slump. The highest sedimentation rates were found in the shallowest samples of the sector, as well as in one sample located in the 485-meter isobath (Sample 6630, $\left.25^{\circ} 49.20^{\prime} \mathrm{S}-45^{\circ} 17.88^{\prime} \mathrm{W}\right)$.

\section{DISCUSSION}

\section{SEDIMENTARY PROCESSES}

As a rule, the Southwestern Atlantic upper margin is marked by low sedimentation rates. Nevertheless, when compared with its SE counterpart, the South African margin, marked by intense upwelling and terrigenous input (Herbert and Compton 2007), the pattern observed in our study area shows important similarities, such as the presence of a starving surface on the outer shelf (Compton and Wiltshire 2009, Compton et al. 2010). In the Holocene mudbelt of the western margin of South Africa, Herbert and Compton (2007) estimated sedimentation rate values ranging from 25 to $240 \mathrm{~cm} \cdot \mathrm{kyr}^{-1}$, whereas on the slope, the values varied from 4 to $22 \mathrm{~cm} . \mathrm{kyr}^{-1}$.

The sedimentation rates calculated here never exceeded the limit of $70 \mathrm{~cm} \cdot \mathrm{kyr}^{-1}$, and estimates from the Coast sector have the same order of magnitude than those from the Inner and Middle Shelf sector (Fig. 2). Outer Shelf/Upper Slope sector sediments present sedimentation rate values that are negligible, indicating the relict character of part of this sector.

Cores from the Coast sector also exhibit several age inversions, which may represent both sediment reworking due to hydrodynamic factors or biological activity. This aspect has been previously analyzed in the scientific literature (Kinoshita et al. 2002, Wood et al. 2006).

The highest sedimentation rates on the shelf were found on the Cabo Frio (around $23^{\circ} \mathrm{S}$ ) and Santa Catarina (around $27^{\circ} \mathrm{S}$ ) upwelling zones, which may be an indication that the marine productivity matter may represent a significant process on the sedimentary processes. On the other hand, these two areas are located closer to the main potential allochthonous sources of terrigenous sediments in the area, e.g., the Paraíba do Sul and La Plata rivers.

Figure 2 also shows a marked latitudinal break at $25^{\circ} \mathrm{S}$, with an abrupt decrease in sedimentation rates northward to São Sebastião Island. In fact, this break marks the limit of influence of the sediments originating from the Río de La Plata as stated by Campos et al. (2008) and Mahiques et al. (2008).

There is a significant difference in terms of sedimentation rates when comparing the Middle Shelf sector with the Outer Shelf/Upper Slope sector sediments. Figure 3 shows a cross-shelf shallow seismic (chirp) profile in which it is possible to recognize a marked break in the sedimentary processes. The sea bottom below the 140-meter isobaths is marked by a strong roughness that, in conjunction with the low sedimentation rates obtained, indicates the effectiveness of the Brazil Current (BC) moving over the outer shelf and upper slope in reworking sediments (Silveira et al. 2001). This aspect has been locally pointed out in previous papers (Macario et al. 2004, Mahiques et al. 2007) but, considering our data, it acquires a regional importance that extends all along the outer shelf and upper slope, between $23^{\circ} \mathrm{S}$ and $27^{\circ} \mathrm{S}$.

A representative cross-margin transect of current speeds is shown in Figure 4. The strong correlation between both speed and sedimentation rate can be seen. In this sense, the slight increase in sedimentation rate at the 450- and 500-meter isobaths is coincident with the transition between the southward flow of the Brazil Current (BC) and the northward flow of the Intermediate Western Boundary Current.

Evidence of Sea Level Stabilizations Prior to the Mid-Holocene MaXimum

The scarcity of datings and reliable sea level indicators is the main characteristic of the sea level curves of the southern Brazilian shelf prior to 7,000 cal yr BP. Most of the papers published on the subject is restricted to correlations of the morphosedimentary features such as submerged terraces with global sea level curves. The study by Correa (1996) is one of the exceptions and, according to this report, stabilization periods occurred at 9,000 cal yr BP (between 32 and 45 meters below sea level) (mbsl) and 8,000 cal yr BP (between 20 and $25 \mathrm{mbsl}$ ). We found some indication of these paleo-sea levels. A core collected on the coast off São Sebastião (SS1, see Table I) represents a sequence of sediments varying from a mixohalyne environment, dated at 39000 cal yr BP (organic matter and benthic foraminifers) at 

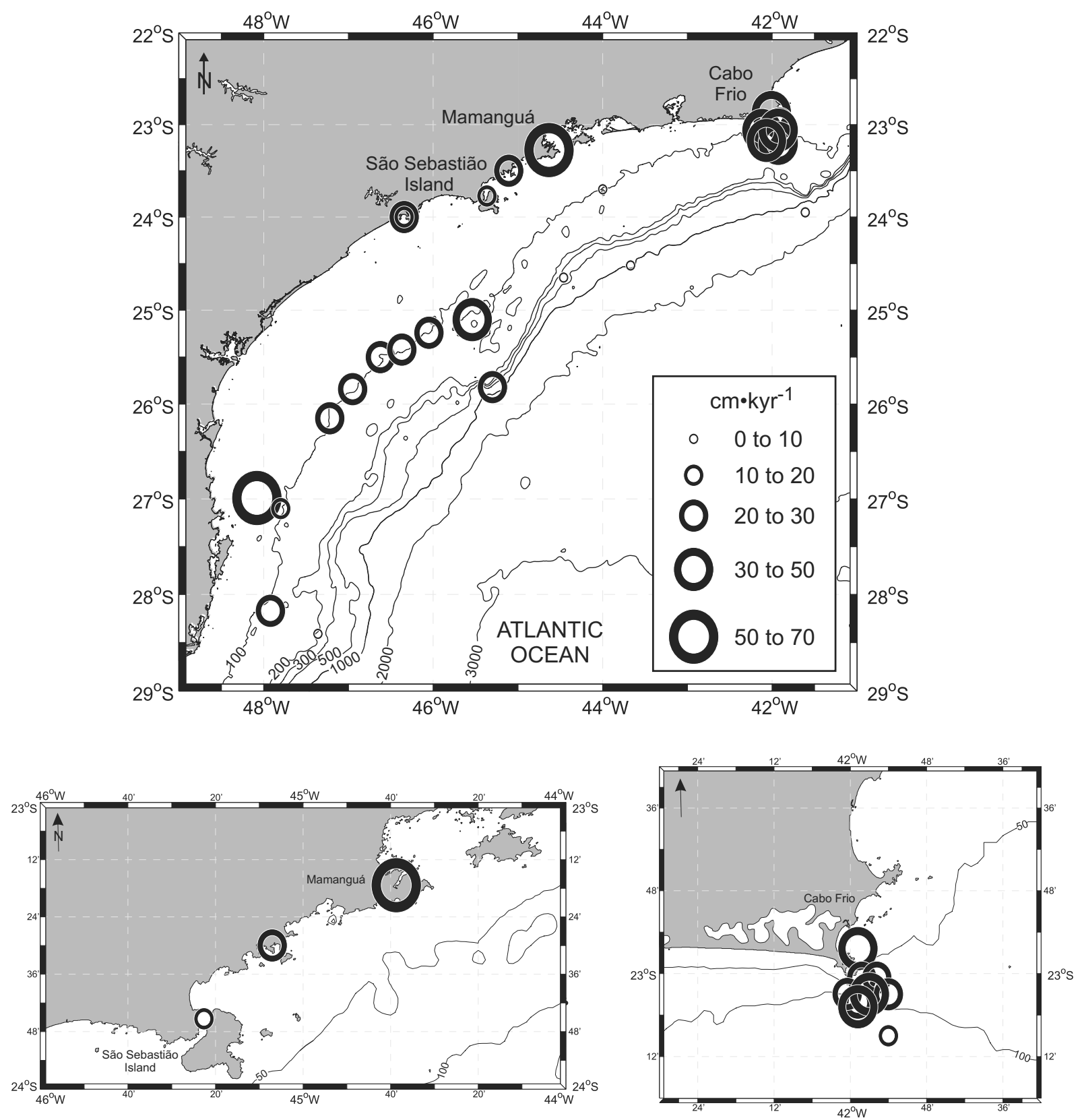

Fig. 2 - Average sedimentation rate values (in $\mathrm{cm}^{\mathrm{kyr}}{ }^{-1}$ ) of selected samples. See the Methods section for the criteria for the choice of the samples used to determine sedimentation rates.

$300 \mathrm{~cm}$ (6 meters bmsl) to freshwater sediments at $136 \mathrm{~cm}$ dated at $10240 \pm 40 \mathrm{yr}$ BP. This depth also marks an erosional contact, and sediments located above it exhibit properties of the Mid- to Late-Holocene ages. Due to the location of the core at the present water depth of $3 \mathrm{mbsl}$, this evidence of a coastal environment in such shallow waters during the Isotope Stage 3 rep- resents a paradox when the worldwide sea level curves are considered. In this sense, one hypothesis to explain these anomalous ages would be a general contamination of organic matter originally deposited during the Isotope Stage 5e by young carbon (Hanebuth et al. 2006). On the other hand, the coherency of the data along the core is noteworthy. These data do not represent the 


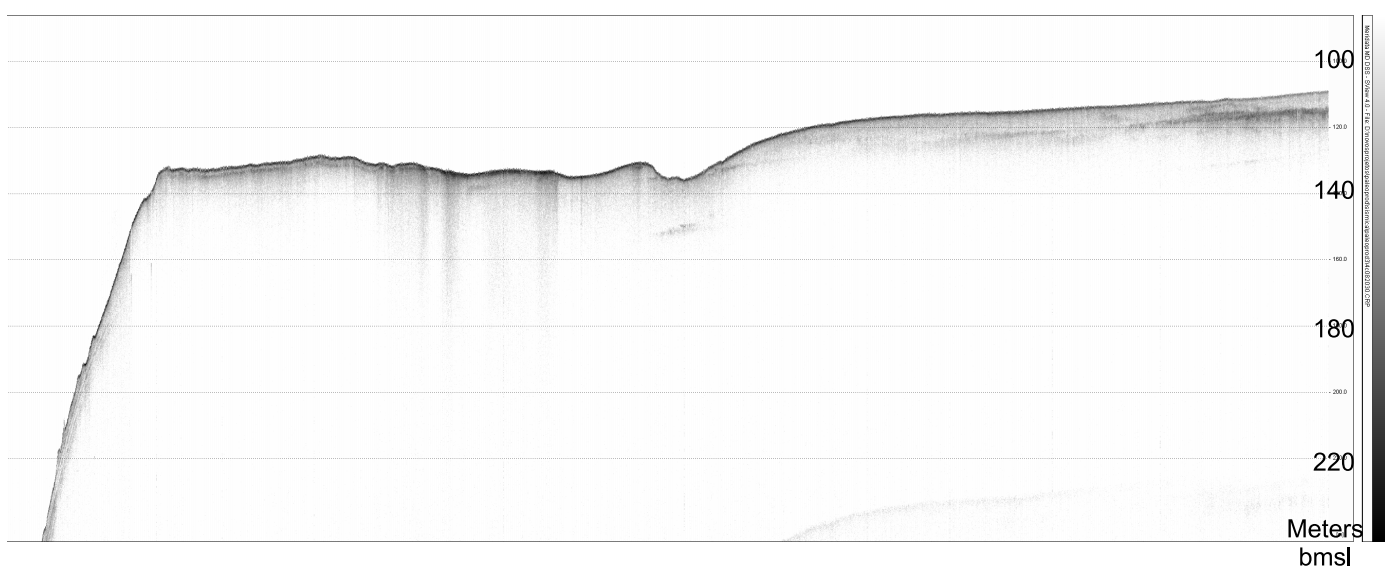

$52 \mathrm{~km}$

Fig. 3 - Cross-shelf shallow seismic (2-8 kHz chirp) profile showing a marked break in the bottom morphology and echo character at $130 \mathrm{mbsl}$.

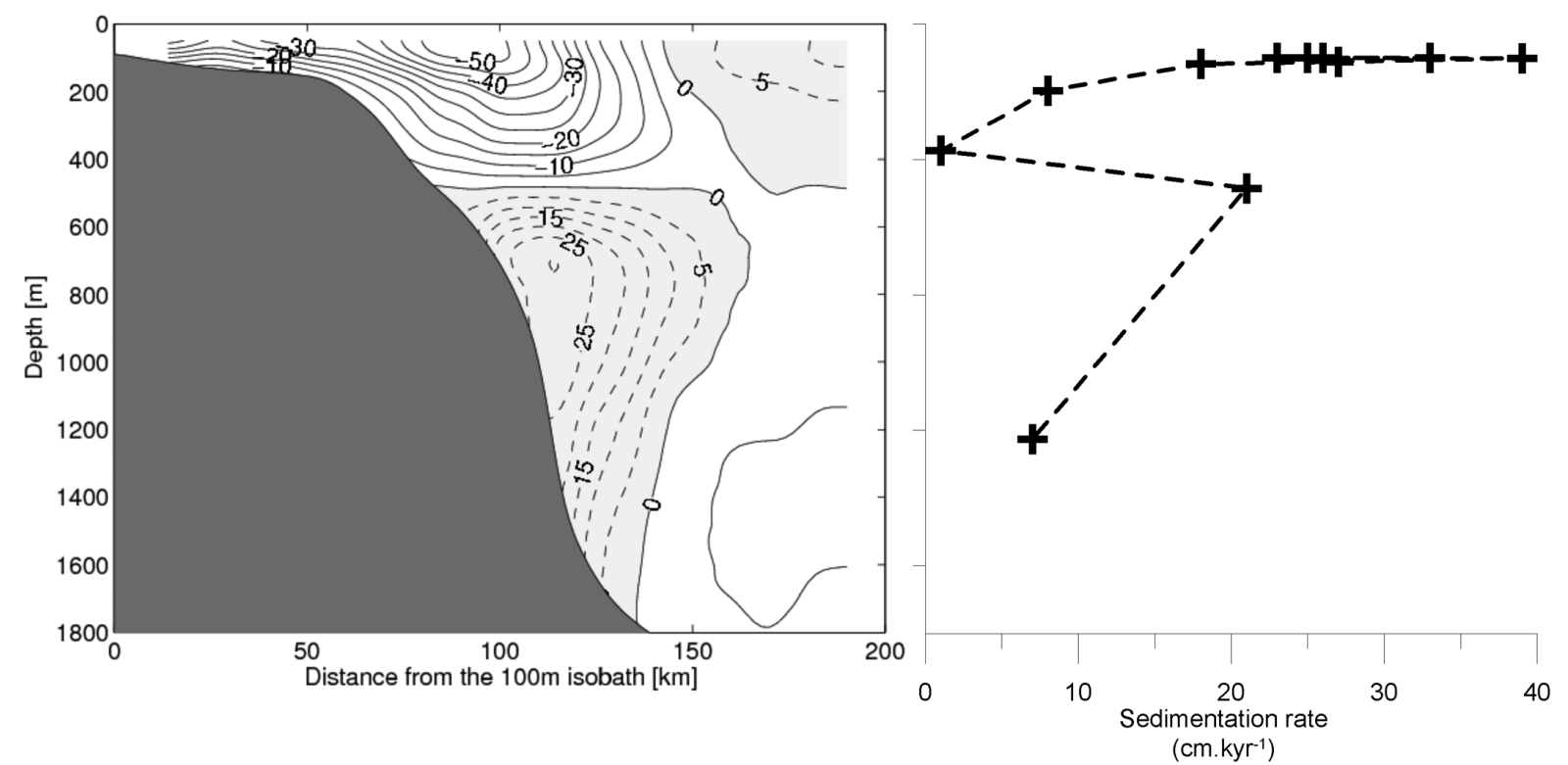

Fig. 4-(Left) Vertical section of a typical absolute geostrophic velocity pattern (in cm/s) off the São Sebastião Island $\left(\sim 24.5-25.5^{\circ} \mathrm{S}\right)$. Measurements were taken in September 2003. (b) Bathymetrical variation of the average sedimentation rates (in $\mathrm{cm} . \mathrm{kyr}^{-1}$ ).

first evidence of high sea level during the Isotope Stage 3; in fact, other indications of sea level highstands of the same age have been found worldwide (Mausz and Hassler 2000, Rodriguez et al. 2000, Hanebuth et al. 2006, Angulo et al. 2008, among others). Nevertheless, further work is needed to improve our understanding of the significance of these data.

Sedimentological evidence of past sea levels was also found in cores FLT01 (Fig. 5) and 7616 (see Tables I and II for further information). Both cores exhibit a passage from sandy beach sediments, attributed to intense bioturbation from Callichirus major (Crustacea, Thalassinidea), to sandy mud sediments. In core 7616, located at $100 \mathrm{mbsl}$, the top of the sandy facies was dated at $12170 \pm 70 \mathrm{yr}$ BP (beyond the limit of calibration). In core FLT01, collected at a water depth of $3 \mathrm{mbsl}$, the top of the facies was dated at $7470 \pm 60 \mathrm{yr}$ BP (7734-8116 cal yr BP), as previously reported by Mahiques and Souza (1999).

Finally, a beach rock located at 13 mbsl presented 


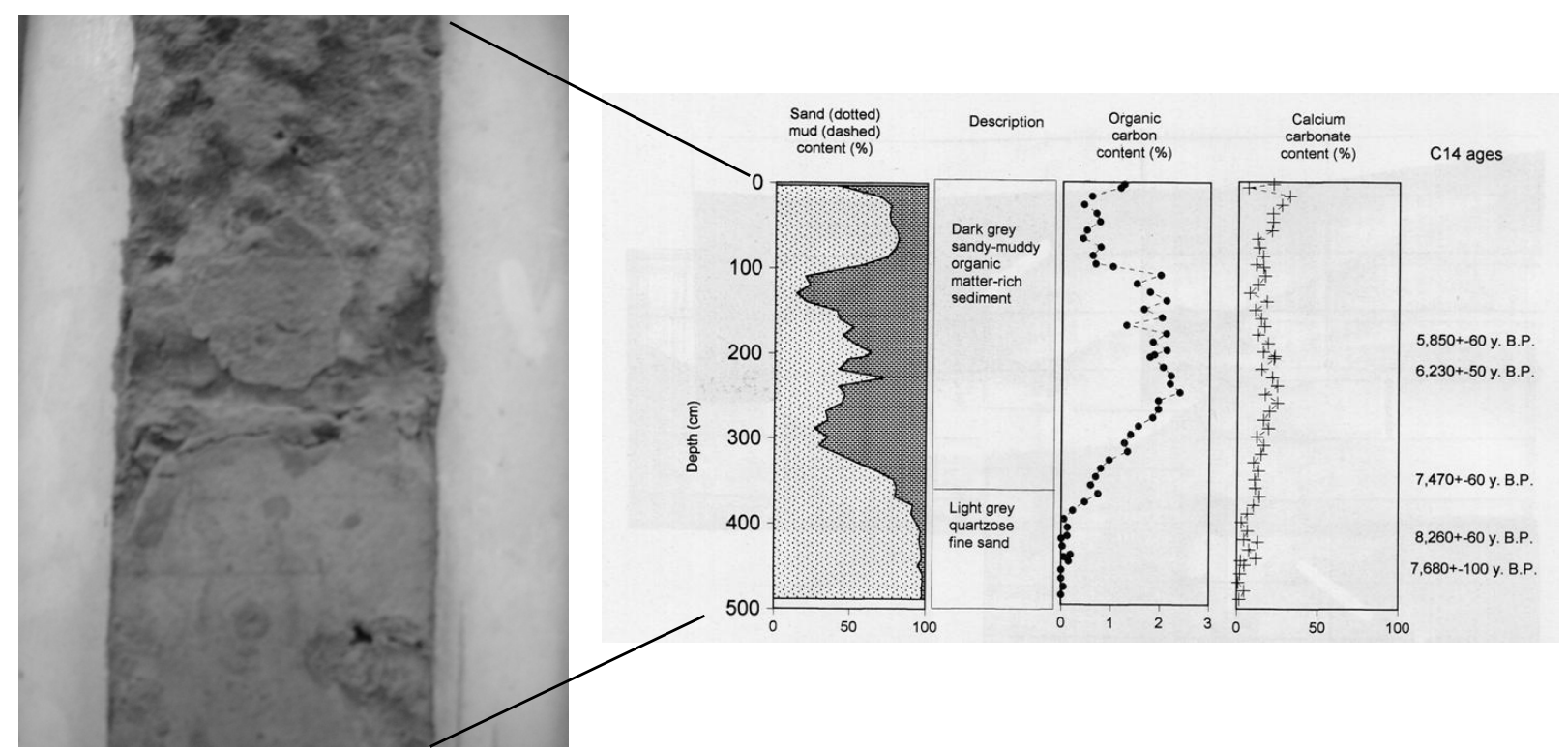

Fig. 5 - Transition between intertidal (below) to infralitoral sediments in a sediment core from the northern coast of the São Paulo Bight. See Mahiques and Souza (1999) for details.

a consistent set of four datings obtained from different materials (total rock, bivalve and fecal pellets), presenting an average age of $8470 \pm 110$ cal yr BP (Fig. 6).

Although the assumption that beach rocks represent the sea level during the time of their formation may not be valid (Kelletat 2006), these data may indicate the minimum depth of a sea level stabilization that occurred immediately before the Melt Water Pulse I-C, as reported in the scientific literature (Clark et al. 2001).

\section{CONCLUSIONS}

In this paper we summarize all of the information on radiocarbon datings compiled by the authors in the São Paulo Bight (southern Brazilian upper margin). Our results confirm the strong dependency of the dynamics of the shelf current system, as well as those of the Brazil Current-Intermediate Western Boundary Current (BCIWBC) system, in the sedimentary processes of the area.

The sediments from the Coast sector exhibit sedimentation rates that may vary from 12 to $68 \mathrm{~cm} \cdot \mathrm{kyr}^{-1}$. Several cores from this data set exhibit characteristics of reworking and/or bioturbation, as has been previously observed by other authors.

The sediments from the Inner and Middle Shelf sector present rates that are equivalent to those of the
Coast. The highest values of sedimentation rates were found in the zones that are more favorable for upwelling processes, suggesting that organic production may act as an important source for particulate sediments. Nevertheless, the proximity of these areas to the main source of terrigenous input must not be neglected.

The sediments from the Outer Shelf/Upper Slope sector are those that are directly affected by the BCIWBC system. As a rule, there is a clear relationship between current speeds and sedimentation rates. A Transition Zone between the cores of these two main flows is also recognizable in the sedimentation rate values.

At least three indicators of the paleo sea level at $12200 \mathrm{yr}$ BP (conventional radiocarbon age) (103 mbsl), 8300-8800 cal yr BP (13 mbsl) and 7700-8100 cal yr BP (6 mbsl) were found. A Marine Isotope Stage 3 highstand at $6 \mathrm{mbsl}$ found in the coast off the São Paulo State will require further study for confirmation.

\section{ACKNOWLEDGMENTS}

This paper is a contribution to the IGCP526 Project (Risks, Resources, Record of the Past on the Continental Shelf). It also represents a contribution from the researchers of the Institute of Oceanography of the University of São Paulo, to the Brazilian Program of Ma- 


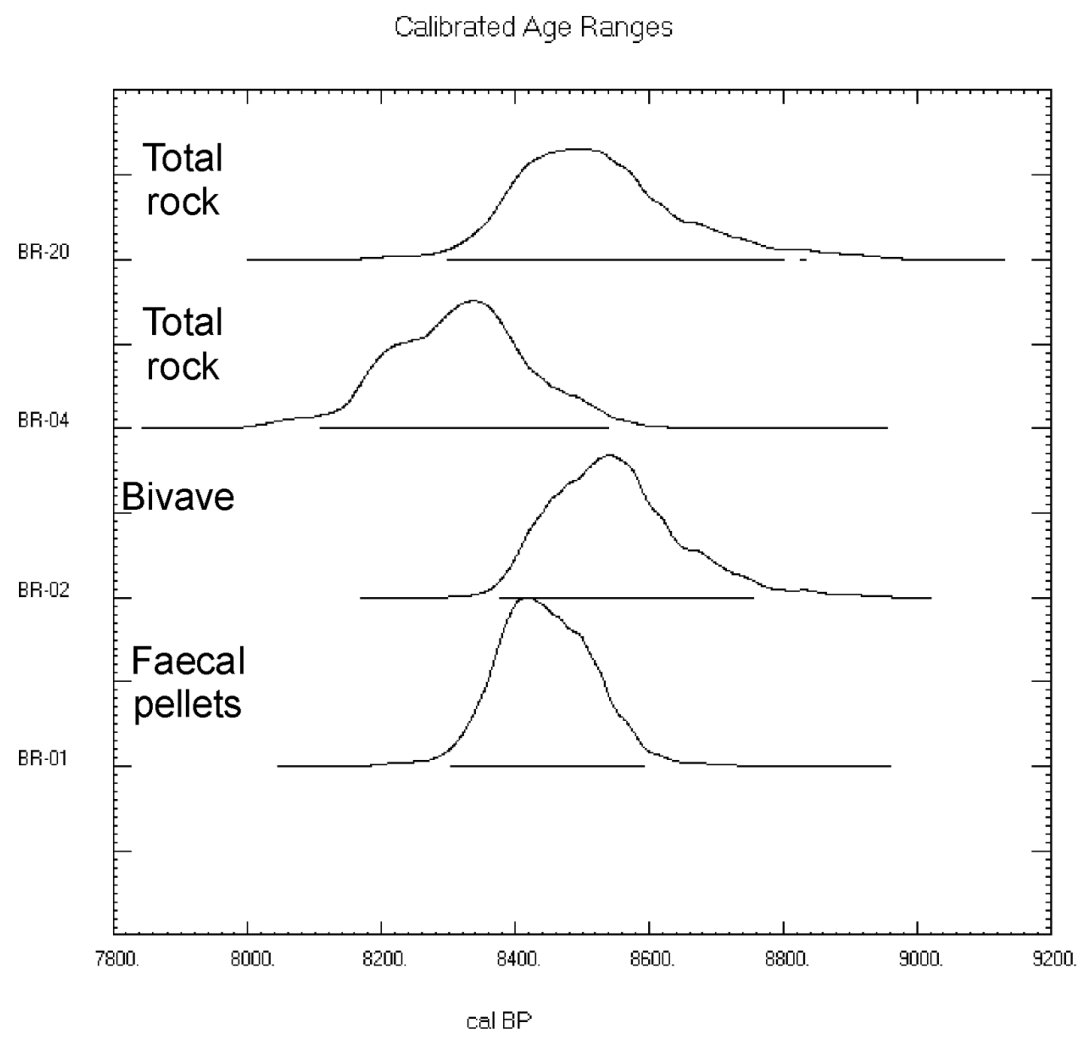

Fig. 6 - Calibrated age ranges of four samples from a beach rock located at $13 \mathrm{mbsl}$ on the northern coast of São Paulo Bight. The average age of $8470 \pm 110$ cal BP is consistent with a phase of sea level stabilization occurring prior to the Melt Water Pulse I-C (Clark et al. 2001).

rine Geology and Geophysics (PGGM). Financial support was provided by Fundação de Amparo à Pesquisa do Estado de São Paulo (FAPESP) (grants 03/107400 and 07/54657-0) and Conselho Nacional de Desenvolvimento Científico e Tecnológico (CNPq) (300593/ 2007-4).

\section{RESUMO}

O objetivo deste trabalho foi a geração de um inventário dos dados de datação de radiocarbono obtidos de sedimentos do Embaiamento de São Paulo (Margem Continental Superior do Sul do Brasil) e analisar os dados em termos de processos sedimentares quaternários e taxas de sedimentação. Um total de 238 datações ao radiocarbono de materiais coletados com a utilização de procedimentos amostrais diferentes foi considerado neste trabalho. As taxas de sedimentação variaram de menor que 2 a $68 \mathrm{~cm} \cdot \mathrm{kyr}^{-1}$. As taxas de sedimentação mais altas foram encontradas em um sistema costeiro de baixa energia (tipo ría), bem como nas zonas de ressurgência de Santa Catarina e Cabo Frio. As taxas mais baixas foram encon- tradas na plataforma externa e talude superior. Nossos resultados confirmam a forte dependência do sistema de correntes de plataforma, com ênfase no aporte terrígeno oriundo do Rio da Prata, transportado através da Corrente Costeira do Brasil, e da dinâmica da Corrente do Brasil - Corrente de Contorno Intermediária (CB-CCI) nos processos sedimentares. Pelo menos três indicadores de paleo-níveis marinhos foram encontrados a 12200 anos AP (idade radiocarbônica convencional) (103 metros abaixo do nível do mar atual - manm), 8300-8800 cal anos AP (13 manm) e 7700-8100 cal anos BP (6 manm).

Palavras-chave: margem continental, quaternário, radiocarbono, nível do mar, sedimentação.

\section{REFERENCES}

Angulo RJ, Souza MC, Assine Ml, Pessenda LCR AND DISARÓ ST. 2008. Chronostratigraphy and radiocarbon age inversion in the Holocene regressive barrier of Paraná, southern Brazil. Mar Geol 252: 111-119.

Angulo RJ, Souza MC, ReImer PJ And SASAOKA SK. 2005. Reservoir effect of the southern and souheastern Brazilian coast. Radiocarbon 47: 67-73. 
CAmpos EJd, Mulkherjee S, Piola AR and CARvaLHO FMS. 2008. A note on a mineralogical analysis of the sediments associated with the Plata River and Patos Lagoon outflows. Cont Shelf Res 28: 1687-1691.

Carroll M, Kowalewski M, Simoes MG and GoodFRIEND GA. 2003. Quantitative estimates of time-averaging in terebratulid brachiopod shell accumulations from a modern tropical shelf. Paleobiology 29: 381-402.

Clark PU, Marshall SJ, Clarke GKC, Hostetler SW, LiCCIARdi JM AND TELLER JT. 2001. Freshwater forcing of abrupt climate change during the last Glaciation. Science 293: 283-287.

COMpton JS AND Herbert CT, HofFMAn MT, SCHNeIDER RR AND STUUT JB. 2010. A tenfold increase in the Orange River mean Holocene mud flux: implications for soil erosion in South Africa. The Holocene 20: 115-122. doi: $10.1177 / 0959683609348860$.

Compton JS And Wiltshire JG. 2009. Terrigenous sediment export from the western margin of South Africa on glacial to interglacial cycles. Mar Geol 266: 212-222.

CORREA ICS. 1996. Les variations du niveau de la mer durant les derniers 17.500 ans BP: l'exemple de la plateforme continentale du Rio Grande do Sul - Brésil. Mar Geol 130: 163-178.

Eastoe CJ, Fish S, Fish P, Gaspar MD and Long A. 2002. Reservoir corrections for marine samples from the South Atlantic coast, Santa Catarina state, Brazil. Radiocarbon 44: 145-148.

Figueira RCL, Tessler MG And Mahiques MM. 2007. Is there a technique for the determination of sedimentation rates based on calcium carbonate content? A comparative study on the southeastern Brazilian shelf. Soils Found 47: 649-656.

Furtado VV, Bonetti Filho J And Conti LA. 1996. Paleo river morphology and sea level changes at southeastern Brazilian continental shelf. An Acad Bras Cienc 68: 163-169.

Hanebuth TJJ, Saito Y, Tanabe S, Vu QL and NGo QT. 2006. Sea levels during late marine isotope stage 3 (or older?) reported from the Red River delta (northern Vietnam) and adjacent regions. Quat Int 145-146: 119134.

Haug G, Hughen Ka, Sigman D, Peterson L and RoHL U. 2001. Southward migration of the Intertropical Convergence Zone through the Holocene. Science 293: 1304-1308.

Herbert CT AND COMPton J. 2007. Geochronology of Holocene sediments on the western margin of South Africa. South Afr Jour Geol 110: 327-338.
Hughen KA ET AL. 2004. Marine04 Marine radiocarbon age calibration, 26-0 ka BP. Radiocarbon 46: 1059-1086.

Kelletat D. 2006. Beachrock as sea-level indicator? Remarks from a geomorphological point of view. Jour Coast Res 22: 1558-1564.

Kinoshita A, Brunetti A, Avelar WEP, Mantelatto FLM, Simões MG, FrANSOZO A AND BAFFA O. 2002. ESR dating of a subfossil Shell from Couve Island, Ubatuba, Brazil. Appl Rad Isot 57: 497-500.

Kowsmann RO And Costa MOA. 1979. Sedimentação quaternária da margem continental brasileira e das áreas oceânicas adjacentes. In: REMAC PROJECT (Final Report). Rio de Janeiro, Petrobras, p. 1-55.

MaCARIo KD, Anjos RM, Gomes PRS, UEIREDo JR AG, LACERda de Souza C, BArbosa CF, CoImbra MM AND ELMORE D. 2004. AMS radiocarbon dating on Campos basin, southeast Brazilian continental slope. Nucl Instr Meth Phys Res B 223-224: 535-539.

Mahiques MM, Bícego MC, Silveira ICA, SousA SHM, LOUREnÇO RA AND FukUmoto MM. 2005. Modern sedimentation in the Cabo Frio upwelling system, Southeastern Brazilian shelf. An Acad Bras Cienc 77: 535-548.

Mahiques MM, Fukumoto MM, Silveira ICA, Figueira RCL, Bícego MC, LOUREnÇO RA AND SousA SHM. 2007. Sedimentary changes on the Southeastern Brazilian upper slope during the last 35,000 years. An Acad Bras Cienc 79: 171-181.

Mahiques MM, Mishima Y AND Rodrigues M. 1999. Characteristics of the sedimentary organic matter on the inner and middle continental shelf between Guanabara Bay and São Francisco do Sul, southeastern Brazilian margin. Cont Shelf Res 19: 775-798.

Mahiques MM, Silveira ICA, Sousa SHM AND RoDRIGUES M. 2002. Post-LGM sedimentation on the outer shelf - upper slope of the northernmost part of the São Paulo Bight, southeastern Brazil. Mar Geol 181: 387400 .

MAHIQUeS MM AND SOUZA LAP. 1999. Shallow seismic reflectors and upper Quaternary sea level changes in the Ubatuba region, São Paulo State, Southeastern Brazil. Rev Bras Oceanogr 47: 1-10.

Mahiques MM, TASsinari CCG, Marcolini S, VioLANTE RA, Figueira RCL, Silveira ICA, Burone L AND SousA SHM. 2008. Nd and Pb isotope signatures on the southeastern South American Upper Margin: Implications for sediment transport and source rocks. Mar Geol 250: 51-63. 
Mahiques MM, Tessler MG, Ciotti AM, Silveira ICA, Sousa SHM, Figueira RCL, TASSINARI CCG, FuRTAdo VV AND PAssos RF. 2004. Hydrodynamically driven patterns of recent sedimentation in the shelf and upper slope off Southeast Brazil. Cont Shelf Res 24: $1685-1697$.

Mahiques MM, Wainer ICK, Burone L, Nagai R, Sousa SHM, Figueira RCL, Silveira ICA, BíCEGo MC, Alves DPV And Hammer O. 2009. A highresolution Holocene record on the Southern Brazilian shelf: Paleoenvironmental implications. Quat Int 206: 52-61.

MaUsz B AND HASsLer U. 2000. Luminescence chronology of Late Pleistocene raised beaches in southern Italy: new data of relative sea-level changes. Mar Geol 170: 187-203.

McCormac FG, Hogg AG, Blackwell PG, Buck CE, Higham TFG AND ReImer PJ. 2004. SHCal04 Southern Hemisphere calibration, 0-11.0 cal kyr BP. Radiocarbon 46: 1087-1092.

MÉndez G AND Vilas F. 2005. Geological antecedents of the Rias Baixas (Galicia, northwest Iberian Peninsula). J Marine Syst 54: 195-207.

Möller Jr OO, Piola AR, Freitas AC And CAmpos EJD. 2008. The effects of river discharge and seasonal winds on the shelf off southeastern South America. Cont Shelf Res 28: 1607-1624.

NADAL DE MASi MA. 1999. Prehistoric hunter-gatherer mobility on the Southern Brazilian coast: Santa Catarina island. Unpublished PhD Dissertation. Stanford University, $186 \mathrm{p}$.

Nagai RH, Sousa SHM, Burone L And Mahiques MM. 2009. Paleoproductivity changes during the Holocene in the inner shelf of Cabo Frio, southeastern Brazilian continental margin: Benthic foraminifera and sedimentological proxies. Quat Int 206: 62-71.
Rocha J, Milliman JD, SANTANa CI ANd ViCAlVi MA. 1975. Southern Brazil. Upper continental margin sedimentation off Brazil. Contr Sedimentol 4: 117-150.

Rodriguez AB, ANDERson JB, BANFIELd LA, TAVIANi M, ABdulah K AND SNOW JN. 2000. Identification of $\mathrm{a}-15 \mathrm{~m}$ middle Wisconsin shoreline on the Texas inner continental shelf. Palaeogeogr Palaeoclimatol Palaeoecol 158: 25-43.

SilveIRA ICA, SCHMIDT ACK, CAMPOS EJD, GodoI SS AND IKEDA Y. 2001. A Corrente do Brasil ao Largo do Sudeste Brasileiro. Rev Bras Oceanogr 48: 171-183.

SouZA RB AND RoBINSON IS. 2004. Lagrangian and satellite observations of the Brazilian Coastal Current. Cont Shelf Res 24: 241-262.

Wood Slb, Krause Jr RA, Kowalewski M, WeHMILlER J AND SimÕES MG. 2006. Aspartic acid racemization dating of Holocene brachiopods and bivalves from the Southern Brazilian shelf, South Atlantic. Quat Res 66: 323-331.

Woodroffe CD, Beech MR And Gagan MK. 2003. Mid-Late Holocene El Niño variability in the equatorial Pacific from coral microatolls. Geophys Res Lett 30: 1358-1361. doi: 10.1029/2002GL015868, 2003.

ZEMBRUSCKI SG. 1979. Geomorfologia da margem continental sul brasileira e das bacias oceânicas adjacentes. In: CHAVES HAF (Ed), Geomorfologia da margem continental brasileira e áreas oceânicas adjacentes. REMAC Project Series, Rio de Janeiro, Petrobras 7: 129-177. 